\title{
Weighted Recognizability over Infinite Alphabets
}

\author{
Maria Pittou $^{a}$ and George Rahonis ${ }^{a}$
}

Dedicated to the memory of Zoltán Ésik

\begin{abstract}
We introduce weighted variable automata over infinite alphabets and commutative semirings. We prove that the class of their behaviors is closed under sum, and under scalar, Hadamard, Cauchy, and shuffle products, as well as star operation. Furthermore, we consider rational series over infinite alphabets and we state a Kleene-Schützenberger theorem. We introduce a weighted monadic second order logic and a weighted linear dynamic logic over infinite alphabets and investigate their relation to weighted variable automata. An application of our theory, to series over the Boolean semiring, concludes to new results for the class of languages accepted by variable automata.
\end{abstract}

Keywords: infinite alphabets, semirings, weighted variable automata, weighted $M S O$, weighted $L D L$

\section{Introduction}

The last two decades a large body of research has been devoted to the development of models for infinite state systems which have finite control structure and handle data from an unbounded domain. This research led to the concept of finite automata over infinite alphabets. Motivating examples for such models consist, for instance, XML schemas, software with integer parameters, and system specification and verification. Later on, it came up that finite automata over infinite alphabets can contribute also to a series of interesting topics namely, the problem of query graph databases [33], reasoning about systems with resource generation capabilities $[10,11]$, learning theories [30], and systems with freshness needed in object-oriented languages and security protocols $[6]$.

Several models of automata with data values, i.e., over infinite alphabets have been investigated, namely register [24, 28, 29, 37], data [5], pebble [28, 36, 39], nominal [10], variable [21, 22], and $P$ automata [9]. All these models refer to qualitative aspects of infinite state systems. Furthermore, rational [1, 25] and logic definable languages $[4,36]$ have been studied over infinite alphabets.

\footnotetext{
${ }^{a}$ Department of Mathematics, Aristotle University of Thessaloniki, 54124 Thessaloniki, Greece. E-mail: \{mpittou, grahonis\}@math.auth.gr
} 
In this paper we intend to study automata models over infinite alphabets in the quantitative setup. Our motivation origins from the fact that several applications require a quantitative analysis of systems, for instance the resource usage control where resource variables are mapped to infinite domains [10,11]. It is well-known that weighted automata is a reasonable tool for the description of quantitative features of computing systems [14]. According to our best knowledge, a quantitative counterpart for automata over infinite alphabets does not exist. In [8] the authors considered quantitative infinite alphabets to model controlled variables for the controller synthesis problem from incompatible situations. For our investigation, we chose the concept of variable automata from [21, 22]. Variable automata are simple in their definition and implementation in contrast to other proposed models. Despite their simplicity, variable automata and their extensions appeared to be expressive enough for several applications. Indeed, in [2] the authors introduced fresh variable automata to describe web services in which the agents exchange data ranging over infinite domains. Furthermore, in [3], fresh variable automata were equipped with guards consisting of equalities and disequalities. In [10] variable automata were extended to consume data words, in order to express security policies (safety properties) for model checking programs that dynamically generate and operate over resources. Very recently, variable automata have been also used for querying graph databases [43]. In a similar approach, a variable $L T L$ was has been investigated in [23]. More precisely, the atomic propositions in that logic were parameterized with variables over some finite or infinite domain in order to express specifications over large, possibly infinite domains. The model checking problem has been also studied for that setting (cf. also [38]).

We consider our weighted variable automata over an infinite alphabet $\Sigma$ and a commutative semiring $K$, and provide a systematic study of the class of their behaviors. Our framework builds upon the techniques which were developed in $[26,27]$ for variable tree automata over infinite ranked alphabets. We prove that, if in addition the semiring $K$ is idempotent, then the class of series accepted by our models is closed under sum, and scalar, Hadamard, Cauchy, and shuffle products, as well as under star operation. As we indicate by a simple example, the proofs for the aforementioned properties require new techniques than the well-known ones for recognizable series [14]. We define rational series over infinite alphabets and state a Kleene-Schützenberger type theorem. Furthermore, we introduce a weighted monadic second order logic and a weighted linear dynamic logic over infinite alphabets. We show the expressive equivalence of the latter logic to our weighed automata, whereas the corresponding equivalence requires fragments on the weighted monadic second order logic. Therefore, several well-known results from classical weighted automata theory hold also for our weighted automata over infinite alphabets. Moreover, by considering the Boolean semiring $\mathbb{B}$, we derive as an application of our theory new results for the class of variable automata of [21, 22]. This shows the robustness of our theory and the theory of variable automata [21, 22].

Apart from this Introduction, the paper contains 7 sections. In Section 2 we present some preliminary background. In Section 3 we introduce our weighted variable automata and in Section 4 we establish the closure properties of the class 
of series accepted by our models. Then, in Section 5 we consider rational series over infinite alphabets and state our Kleene-Schützenberger theorem. Sections 6 and 7, respectively are devoted to weighted monadic second order logic and weighted linear dynamic logic, and their relation to weighted variable automata. In Section 8 we expose the new results on variable automata derived by our theory. Finally, in the Conclusion, we present some ideas for future research.

A preliminary version of this paper appeared in [32] (cf. also [31]).

\section{Preliminaries}

Let $\Sigma$ be an alphabet, i.e., a nonempty (potentially infinite) set. As usually, we denote by $\Sigma^{*}$ the set of all finite words over $\Sigma$ and $\Sigma^{+}=\Sigma^{*} \backslash\{\varepsilon\}$, where $\varepsilon$ is the empty word. A subset $L \subseteq \Sigma^{*}$ is a language over $\Sigma$. A word $w=\sigma_{0} \ldots \sigma_{n-1}$, where $\sigma_{0}, \ldots, \sigma_{n-1} \in \Sigma(n \geq 1)$, is written also as $w=w(0) \ldots w(n-1)$ where $w(i)=\sigma_{i}$ for every $0 \leq i \leq n-1$. For every finite word $w=w(0) \ldots w(n-1)$ and every $0 \leq i \leq n-1$ we denote by $w>i$ the suffix $w(i) \ldots w(n-1)$. If $S$ is a set, then $\mathcal{P}(S)$ will denote the powerset of $S$, and the notation $S^{\prime} \subseteq_{\text {fin }} S$ means that $S^{\prime}$ is a finite subset of $S$.

A semiring $(K,+, \cdot, 0,1)$ is an algebraic structure such that $(K,+, 0)$ is a commutative monoid, $(K, \cdot, 1)$ is a monoid, $0 \neq 1$, is both left- and right-distributive over + , and $0 \cdot k=k \cdot 0=0$ for every $k \in K$. If no confusion arises, we shall denote the semiring simply by $K$ and the - operation simply by concatenation. The semiring $K$ is called commutative if the monoid $(K, \cdot 1)$ is commutative. Moreover, $K$ is called additively idempotent (or simply idempotent), if $k+k=k$ for every $k \in K$. Finally, $K$ is called locally finite if every finitely generated subsemiring is finite. Interesting examples of semirings are the following:

- the semiring $(\mathbb{N},+, \cdot, 0,1)$ of natural numbers,

- the Boolean semiring $\mathbb{B}=(\{0,1\},+, \cdot, 0,1)$,

- the tropical or min-plus semiring $\left(\mathbb{R}_{+} \cup\{\infty\}\right.$, min, $\left.+, \infty, 0\right)$ where $\mathbb{R}_{+}=\{r \in$ $\mathbb{R} \mid r \geq 0\}$,

- the arctical or max-plus semiring $\left(\mathbb{R}_{+} \cup\{-\infty\}\right.$, max, $\left.+,-\infty, 0\right)$,

- the Viterbi semiring $([0,1], \max , \cdot, 0,1)$,

- every bounded distributive lattice with the operations supremum and infimum, and especially the fuzzy semiring $F=([0,1]$, max, min, 0,1$)$.

All the above semirings, except the first one, are idempotent.

Let $\Sigma$ be an alphabet and $K$ a semiring. A formal series (or simply series) over $\Sigma$ and $K$ is a mapping $s: \Sigma^{*} \rightarrow K$. For every $w \in \Sigma^{*}$ we write $(s, w)$ for the value $s(w)$ and refer to it as the coefficient of $s$ on $w$. The support of $s$ is 
the set $\operatorname{supp}(s)=\left\{w \in \Sigma^{*} \mid(s, w) \neq 0\right\}$. A series with finite support is called a polynomial. The constant series $\widetilde{k}(k \in K)$ is defined, for every $w \in \Sigma^{*}$, by $(\widetilde{k}, w)=k$. Moreover, for every $w \in \Sigma^{*}$, we denote by $\bar{w}$ the series determined, for every $u \in \Sigma^{*}$, by $(\bar{w}, u)=1$ if $u=w$ and 0 , otherwise. The class of all series over $\Sigma$ and $K$ is denoted as usual by $K\left\langle\left\langle\Sigma^{*}\right\rangle\right\rangle$, and the class of polynomials over $\Sigma$ and $K$ by $K\left\langle\Sigma^{*}\right\rangle$. The characteristic series $1_{L} \in K\left\langle\left\langle\Sigma^{*}\right\rangle\right\rangle$ of a language $L \subseteq \Sigma^{*}$ is defined by $\left(1_{L}, w\right)=1$ if $w \in L$ and $\left(1_{L}, w\right)=0$ otherwise.

Let $s, r \in K\left\langle\left\langle\Sigma^{*}\right\rangle\right\rangle$ and $k \in K$. The sum $s+r$, the scalar products $k s$ and $s k$ as well as the Hadamard product $s \odot r$ are defined elementwise by $(s+$ $r, w)=(s, w)+(r, w),(k s, w)=k \cdot(s, w),(s k, w)=(s, w) \cdot k$, and $(s \odot r, w)=$ $(s, w) \cdot(r, w)$, respectively, for every $w \in \Sigma^{*}$. It is well-known that the structure $\left(K\left\langle\left\langle\Sigma^{*}\right\rangle\right\rangle,+, \odot, \widetilde{0}, \widetilde{1}\right)$ is a semiring. Moreover, if $K$ is commutative (resp. idempotent), then $\left(K\left\langle\left\langle\Sigma^{*}\right\rangle\right\rangle,+, \odot, \widetilde{0}, \widetilde{1}\right)$ is commutative (resp. idempotent).

The Cauchy product of $r$ and $s$ is the series $r \cdot s \in K\left\langle\left\langle\Sigma^{*}\right\rangle\right\rangle$ defined for every $w \in \Sigma^{*}$ by

$$
(r \cdot s, w)=\sum_{\substack{u, v \in \Sigma^{*} \\ w=u v}}((r, u) \cdot(s, v))
$$

The $n$ th-iteration $r^{n} \in K\left\langle\left\langle\Sigma^{*}\right\rangle\right\rangle(n \geq 0)$ of a series $r \in K\left\langle\left\langle\Sigma^{*}\right\rangle\right\rangle$ is defined inductively by

$$
r^{0}=\bar{\varepsilon} \quad \text { and } \quad r^{n+1}=r \cdot r^{n} \text { for } n \geq 0 .
$$

Then, we have $\left(r^{n}, w\right)=\sum_{u_{1}, \ldots, u_{n} \in \Sigma^{*}}\left(\left(r, u_{1}\right) \cdot \ldots \cdot\left(r, u_{n}\right)\right)$ for every $w \in \Sigma^{*}$. A series $r \in K\left\langle\left\langle\Sigma^{*}\right\rangle\right\rangle$ is called proper whenever $(r, \varepsilon)=0$. If $r$ is proper, then for every $w \in \Sigma^{*}$ and $\left.n\right\rangle|w|$ we have $\left(r^{n}, w\right)=0$. The star $r^{*} \in K\left\langle\left\langle\Sigma^{*}\right\rangle\right\rangle$ of a proper series $r \in K\left\langle\left\langle\Sigma^{*}\right\rangle\right\rangle$ is defined by $r^{*}=\sum_{n \geq 0} r^{n}$. Thus, for every $w \in \Sigma^{*}$ we have $\left(r^{*}, w\right)=\sum_{0 \leq n \leq|w|}\left(r^{n}, w\right)$

Finally, the shuffle product of $r$ and $s$ is the series $r ш s \in K\left\langle\left\langle\Sigma^{*}\right\rangle\right\rangle$ defined for every $w \in \Sigma^{*}$ by

$$
(r \amalg s, w)=\sum_{\substack{u, v \in \Sigma^{*} \\ w \in u \amalg v}}((r, u) \cdot(s, v))
$$

where $u \amalg v$ denotes the shuffle product of $u$ and $v$.

Next we turn to weighted automata. For this we assume the alphabet $\Sigma$ to be finite. A weighted automaton over $\Sigma$ and $K$ is a quadruple $A=(Q, i n, w t, t e r)$ where $Q$ is the finite state set, in $Q \rightarrow K$ is the initial distribution, wt: $Q \times \Sigma \times$ $Q \rightarrow K$ is a mapping assigning weights to the transitions of the automaton, and ter $: Q \rightarrow K$ is the final (or terminal) distribution.

Let $w=w(0) \ldots w(n-1) \in \Sigma^{*}$. A path of $A$ over $w$ is a sequence of transitions 
$P_{w}:=\left(\left(q_{i}, w(i), q_{i+1}\right)\right)_{0 \leq i \leq n-1}$. The weight of $P_{w}$ is given by the value

$$
\operatorname{weight}\left(P_{w}\right)=i n\left(q_{0}\right) \cdot \prod_{0 \leq i \leq n-1} w t\left(\left(q_{i}, w(i), q_{i+1}\right)\right) \cdot \operatorname{ter}\left(q_{n}\right) .
$$

The behavior of $A$ is the series $\|A\|: \Sigma^{*} \rightarrow K$ whose coefficients are given by

$$
(\|A\|, w)=\sum_{P_{w}} w e i g h t\left(P_{w}\right)
$$

for every $w \in \Sigma^{*}$.

A series $s \in K\left\langle\left\langle\Sigma^{*}\right\rangle\right\rangle$ is called recognizable if $s=\|A\|$ for some weighted automaton $A$ over $\Sigma$ and $K$. As usual we denote by $\operatorname{Rec}(K, \Sigma)$ the class of recognizable series over $\Sigma$ and $K$. Two weighted automata $A=(Q, i n, w t, t e r)$ and $A^{\prime}=\left(Q^{\prime}, i n^{\prime}, w t^{\prime}, t e r^{\prime}\right)$ over $\Sigma$ and $K$ are called equivalent if $\|A\|=\left\|A^{\prime}\right\|$.

Finally, a weighted automaton $A=(Q, i n, w t, t e r)$ over $\Sigma$ and $K$ is called normalized if there exist two states $q_{i n}, q_{t e r} \in Q, q_{i n} \neq q_{t e r}$, such that:

- in $(q)=1$ if $q=q_{i n}$, and $i n(q)=0$ otherwise,

- $\operatorname{ter}(q)=1$ if $q=q_{t e r}$, and $\operatorname{ter}(q)=0$ otherwise, and

- $w t\left(\left(q, \sigma, q_{\text {in }}\right)\right)=w t\left(\left(q_{\text {ter }}, \sigma, q\right)\right)=0$

for every $q \in Q, \sigma \in \Sigma$. We shall denote a normalized weighted automaton $A=$ $(Q, i n, w t, t e r)$ simply by $A=\left(Q, q_{i n}, w t, q_{t e r}\right)$. The next result has been proved by several authors, cf. for instance [18].

Proposition 1. Let $A=(Q, i n, w t, t e r)$ be a weighted automaton over $\Sigma$ and $K$. We can effectively construct a normalized weighted automaton $A^{\prime}$ such that $\left(\left\|A^{\prime}\right\|, w\right)=(\|A\|, w)$ for every $w \in \Sigma^{+}$and $\left(\left\|A^{\prime}\right\|, \varepsilon\right)=0$.

\section{Weighted variable automata}

In this section we introduce the notion of our weighted variable automata. We show that the well-known constructions on weighted automata are not sufficient to obtain the closure properties of the class of series recognized by our models. Therefore, we provide some supplementary matter and we state Lemma 1 which will be needed in the sequel in our constructions.

Let $\Sigma, \Sigma^{\prime}$ be (infinite) alphabets. A relabeling from $\Sigma$ to $\Sigma^{\prime}$ is a mapping $h: \Sigma \rightarrow \mathcal{P}\left(\Sigma^{\prime}\right)$. Next let $\Gamma \subseteq_{\text {fin }} \Sigma, Z$ be a finite set whose elements are called bounded variables and $y$ an element which is called a free variable. We assume that the sets $\Sigma, Z$, and $\{y\}$ are pairwise disjoint. A relabeling $h$ from $\Gamma \cup Z \cup\{y\}$ to $\Sigma$ is called valid if

(i) it is the identity on $\Gamma,{ }^{1}$

\footnotetext{
${ }^{1}$ Abusing notation we identify $\{\sigma\}$ with $\sigma$, for every $\sigma \in \Gamma$.
} 
(ii) $\operatorname{card}(h(z))=1$ for every $z \in Z$,

(iii) $h$ is injective on $Z$ and $\Gamma \cap h(Z)=\emptyset$, and

(iv) $h(y)=\Sigma \backslash(\Gamma \cup h(Z))$.

The above definition means that the application of $h$ on a word $w$ over $\Gamma \cup Z \cup\{y\}$ assigns to every occurrence of a symbol $z \in Z$ in $w$ the same symbol from $\Sigma$, but it is possible to assign different symbols from $\Sigma$ to different occurrences of $y$ in $w$. This justifies the names bounded and free for the set of variables $Z$ and the variable $y$, respectively. It should be clear that a valid relabeling from $\Gamma \cup Z \cup\{y\}$ to $\Sigma$ is well-defined if it is defined only on $Z$ satisfying conditions (ii) and (iii). We shall denote by $V R(\Gamma \cup Z \cup\{y\}, \Sigma)$ the set of all valid relabelings from $\Gamma \cup Z \cup\{y\}$ to $\Sigma$, and simply by $V R(\Gamma \cup Z \cup\{y\})$ if the alphabet $\Sigma$ is understood.

We set $\Delta=\Gamma \cup Z \cup\{y\}$ and let $w \in \Sigma^{*}$. The preimage of $w$ over $\Delta$ is the set $\operatorname{preim}_{\Delta}(w)=\left\{u \in \Delta^{*} \mid\right.$ there exists $h \in V R(\Delta)$ such that $\left.u \in h^{-1}(w)\right\}$.

Now we are ready to introduce our weighted variable automata over the infinite alphabet $\Sigma$ and a semiring $K$.

Definition 1. $A$ weighted variable automaton (wva for short) over $\Sigma$ and $K$ is a pair $\mathcal{A}=\langle\Sigma, A\rangle$ where $\Sigma$ is an infinite alphabet and $A=(Q$, in, wt,ter $)$ is a weighted automaton over $\Gamma_{A}$ and $K$. The input alphabet $\Gamma_{A}$ of $A$ is defined by $\Gamma_{A}=\Sigma_{A} \cup Z \cup\{y\}$, where $\Sigma_{A} \subseteq$ fin $\Sigma, Z$ is a finite alphabet of bounded variables, and $y$ is a free variable. by

The behavior of $\mathcal{A}$ is the series $\|\mathcal{A}\|: \Sigma^{*} \rightarrow K$ whose coefficients are determined

$$
(\|\mathcal{A}\|, w)=\sum_{u \in \text { preim }_{\Gamma_{A}}(w)}(\|A\|, u)
$$

for every $w \in \Sigma^{*}$. Clearly, the above sum is finite and thus $(\|\mathcal{A}\|, w)$ is well-defined for every $w \in \Sigma^{*}$.

Two wva $\mathcal{A}$ and $\mathcal{A}^{\prime}$ over $\Sigma$ and $K$ are called equivalent whenever $\|\mathcal{A}\|=\left\|\mathcal{A}^{\prime}\right\|$.

A series $r$ over $\Sigma$ and $K$ is called $v$-recognizable if there exists a wva $\mathcal{A}$ such that $r=\|\mathcal{A}\|$. We shall denote by $V \operatorname{Rec}(K, \Sigma)$ the class of v-recognizable series over $\Sigma$ and $K$. It should be clear that every weighted automaton $A$ over a subalphabet $\Sigma^{\prime} \subseteq_{\text {fin }} \Sigma$ and $K$ can be considered as a wva such that its transitions labelled by variables carry the weight 0 . Therefore, we get the next result, where the strictness of the inclusion trivially holds by the definition of wva.

Proposition 2. $\bigcup_{\Sigma^{\prime} \subseteq \subseteq_{f i n} \Sigma} \operatorname{Rec}\left(K, \Sigma^{\prime}\right) \subsetneq V \operatorname{Rec}(K, \Sigma)$.

Throughout the paper $\Sigma$ will denote an infinite alphabet, $Z$ a finite set of bounded variables, $y$ a free variable, and $K$ a commutative semiring. In addition, in the present and the next section, $K$ will be assumed to be idempotent. 
In the sequel, we will call a wva $\mathcal{A}=\langle\Sigma, A\rangle$ over $\Sigma$ and $K$, simply a wva.

Definition 2. A wva $\mathcal{A}=\langle\Sigma, A\rangle$ is called normalized if $A$ is normalized.

Proposition 3. Let $\mathcal{A}=\langle\Sigma, A\rangle$ be a wva. We can effectively construct a normalized wva $\mathcal{A}^{\prime}$ such that $\left(\left\|\mathcal{A}^{\prime}\right\|, w\right)=(\|\mathcal{A}\|, w)$ for every $w \in \Sigma^{+}$and $\left(\left\|\mathcal{A}^{\prime}\right\|, \varepsilon\right)=0$.

Proof. We immediately obtain our result by Proposition 1 and Definition 2 .

In the sequel, we wish to investigate closure properties of the class $V \operatorname{Rec}(K, \Sigma)$. For this, we cannot apply the well-known constructions from classical weighted automata theory. For instance, let $\mathcal{A}=\langle\Sigma, A\rangle$ be a normalized wva, where $A=$ $\left(\left\{q_{i n}, q, q_{\text {ter }}\right\}, q_{i n}, w t_{A}, q_{\text {ter }}\right), \Gamma_{A}=\{a\} \cup\{z\} \cup\{y\}$ and transitions with non-zero weights given by $w t_{A}\left(\left(q_{i n}, a, q\right)\right)=w t_{A}\left(\left(q, z, q_{t e r}\right)\right)=1$. Consider also the normalized wva $\mathcal{A}^{\prime}=\left\langle\Sigma, A^{\prime}\right\rangle$ where $A^{\prime}=\left(\left\{q_{\text {in }}^{\prime}, q_{\text {ter }}^{\prime}\right\}, q_{\text {in }}^{\prime}, w t_{A^{\prime}}, q_{\text {ter }}^{\prime}\right), \Gamma_{A^{\prime}}=\left\{a^{\prime}\right\} \cup\left\{z^{\prime}\right\} \cup\left\{y^{\prime}\right\}$ and $w t_{A^{\prime}}\left(\left(q_{i n}^{\prime}, a^{\prime}, q_{t e r}^{\prime}\right)\right)=w t_{A^{\prime}}\left(\left(q_{i n}^{\prime}, y^{\prime}, q_{\text {ter }}^{\prime}\right)\right)=1$. Moreover, let us assume that $a \neq a^{\prime}$. Clearly, $\left(\|\mathcal{A}\|, a a^{\prime}\right)=1$ and $\left(\left\|\mathcal{A}^{\prime}\right\|, a^{\prime}\right)=1$. Nevertheless, if we consider the disjoint union of $A$ and $A^{\prime}$, say the weighted automaton $B$, then $a, a^{\prime} \in \Gamma_{B}$ which implies that we cannot apply a valid relabeling assigning the letter $a^{\prime}$ to $z$. This in turn, implies that the word $a a^{\prime}$ does not belong to the support of the wva derived by the weighted automaton $B$. Furthermore, another problem of this construction is the choice of the free variable among $y$ and $y^{\prime}$ which moreover causes new inconsistencies. Similar, even more complex, situations arise for the constructions of wva proving closure under further properties like Hadamard, Cauchy, and shuffle product. Therefore, we state Lemma 1 below which will be of great importance to our constructions for the closure properties of the class $V \operatorname{Rec}(K, \Sigma)$. We shall need some preliminary matter.

Let $\mathcal{A}=\langle\Sigma, A\rangle$ be a wva where $A=(Q$, in, wt,ter $)$ with $\Gamma_{A}=\Sigma_{A} \cup Z \cup\{y\}$, and $\Sigma^{\prime} \subseteq$ fin $\Sigma$ such that $\Sigma^{\prime} \backslash \Sigma_{A} \neq \emptyset$. We define on $V R\left(\Gamma_{A}\right)$ the relation $\equiv_{\Sigma^{\prime}}$ determined for every $h_{1}, h_{2} \in V R\left(\Gamma_{A}\right)$ by

$$
h_{1} \equiv_{\Sigma^{\prime}} h_{2} \quad \text { iff } \quad h_{1}(\sigma) \cap \Sigma^{\prime}=h_{2}(\sigma) \cap \Sigma^{\prime} \text { for every } \sigma \in Z \cup\{y\} .
$$

It should be clear that $\equiv_{\Sigma^{\prime}}$ is an equivalence relation. Moreover, since $Z \cup\{y\}$ and $\Sigma^{\prime}$ are finite, the index of $\equiv_{\Sigma^{\prime}}$ is finite. Let $V$ be a set of representatives of $V R\left(\Gamma_{A}\right) / \equiv_{\Sigma^{\prime}}$. For every $h \in V$, we let $Z_{h}=\left\{z \in Z \mid h(z) \in \Sigma^{\prime}\right\}$ and $\Gamma_{h}=\Sigma_{A} \cup$ $\Sigma^{\prime} \cup\left(Z \backslash Z_{h}\right) \cup\{y\}$, and we consider the weighted automaton $A_{h}=\left(Q_{h}, i n_{h}, w t_{h}, t e r_{h}\right)$ over $\Gamma_{h}$ and $K$, where $Q_{h}=\left\{q_{h} \mid q \in Q\right\}$ is a copy of $Q, i n_{h}\left(q_{h}\right)=i n(q)$ and $\operatorname{ter}_{h}\left(q_{h}\right)=\operatorname{ter}(q)$ for every $q_{h} \in Q_{h}$. The weight assignment mapping $w t_{h}$ is defined as follows. For every $q_{h}, q_{h}^{\prime} \in Q_{h}, \sigma \in \Gamma_{h}$, we let

$$
w t_{h}\left(\left(q_{h}, \sigma, q_{h}^{\prime}\right)\right)= \begin{cases}w t\left(\left(q, \sigma, q^{\prime}\right)\right) & \text { if } \sigma \in \Sigma_{A} \cup\left(Z \backslash Z_{h}\right) \cup\{y\} \\ w t\left(\left(q, z, q^{\prime}\right)\right) & \text { if } \sigma=h(z) \text { and } z \in Z_{h} \\ w t\left(\left(q, y, q^{\prime}\right)\right) & \text { if } \sigma \in h(y) \cap \Sigma^{\prime} \\ 0 & \text { otherwise }\end{cases}
$$


Without any loss, we assume that the sets $Q_{h}$ are pairwise disjoint. We let $Q_{V}=$ $\bigcup_{h \in V} Q_{h}, \Gamma_{V}=\Sigma_{A} \cup \Sigma^{\prime} \cup Z \cup\{y\}$, and consider the wva $\mathcal{A}_{\left(\Sigma^{\prime}, V\right)}=\left\langle\Sigma, A_{\left(\Sigma^{\prime}, V\right)}\right\rangle$ over $\Sigma$ and $K$, where $A_{\left(\Sigma^{\prime}, V\right)}=\left(Q_{V}, i n_{V}, w t_{V}, t e r_{V}\right)$ is a weighted automaton with input alphabet $\Gamma_{V}$. Its initial and final distribution are defined, respectively, by $i n_{V}(q)=i n_{h}(q), \operatorname{ter}_{V}(q)=\operatorname{ter}_{h}(q)$ for every $q \in Q_{h}, h \in V$. The weight assignment mapping $w t_{V}: Q_{V} \times \Gamma_{V} \times Q_{V} \rightarrow K$ is given by

$$
w t_{V}\left(\left(q, \sigma, q^{\prime}\right)\right)= \begin{cases}w t_{h}\left(\left(q, \sigma, q^{\prime}\right)\right) & \text { if } q, q^{\prime} \in Q_{h} \text { for some } h \in V \\ 0 & \text { otherwise }\end{cases}
$$

for every $q, q^{\prime} \in Q_{V}, \sigma \in \Gamma_{V}$.

Since the weighted automaton $A_{\left(\Sigma^{\prime}, V\right)}$ is the disjoint union of $A_{h}, h \in V$, we get that $\left\|A_{\left(\Sigma^{\prime}, V\right)}\right\|=\sum_{h \in V}\left\|A_{h}\right\|$. Therefore, for every $w \in \Sigma^{*}$, we have

$$
\left(\left\|\mathcal{A}_{\left(\Sigma^{\prime}, V\right)}\right\|, w\right)=\sum_{u \in \text { preimm }}\left(\left\|A_{\Gamma_{V}\left(\Sigma^{\prime}, V\right)}\right\|, u\right)=\sum_{h \in V} \sum_{u \in \text { preim } \Gamma_{h}(w)}\left(\left\|A_{h}\right\|, u\right) .
$$

Lemma 1. $\|\mathcal{A}\|=\left\|\mathcal{A}_{\left(\Sigma^{\prime}, V\right)}\right\|$.

Proof. Let $w=w(0) \ldots w(n-1) \in \Sigma^{*}$. Consider a word $u=u(0) \ldots u(n-1) \in$ $\operatorname{preim}_{\Gamma_{A}}(w)$ and a valid relabeling $h \in V R\left(\Gamma_{A}\right)$ with $w \in h(u)$. We define the word $u^{\prime}=u^{\prime}(0) \ldots u^{\prime}(n-1) \in \Gamma_{V}^{*}$ as follows:

$$
u^{\prime}(i)= \begin{cases}u(i) & \text { if }\left(u(i) \in \Sigma_{A} \cup Z \backslash Z_{h}\right) \text { or }\left(u(i)=y \text { and } w(i) \notin \Sigma^{\prime} \backslash \Sigma_{A}\right) \\ w(i) & \text { if }\left(u(i) \in Z_{h}\right) \text { or }\left(u(i)=y \text { and } w(i) \in \Sigma^{\prime} \backslash \Sigma_{A}\right)\end{cases}
$$

for every $0 \leq i \leq n-1$.

We consider the set of valid relabelings $V^{\prime} \subseteq V$ as follows: $g \in V^{\prime}$ implies that $g(z)=h(z)$ for every $z \in Z_{h} \cap\{u(i) \mid 0 \leq i \leq n-1\}$ and $g(y) \cap \Sigma^{\prime}=h(y) \cap \Sigma^{\prime}$ whenever $u(i)=y$ and $w(i) \in \Sigma^{\prime}$ for some $0 \leq i \leq n-1$. Let $P_{u}^{(A)}$ be a path of $A$ over $u$. Then, by construction of $A_{\left(\Sigma^{\prime}, V\right)}$, for every $g \in V^{\prime}$, there exists a path $P_{u^{\prime}}^{\left(A_{g}\right)}$ of $A_{g}$ over $u^{\prime}$ with weight $\left(P_{u^{\prime}}^{\left(A_{g}\right)}\right)=$ weight $\left(P_{u}^{(A)}\right)$. Clearly, there are $r=\operatorname{card}\left(V^{\prime}\right)$ such paths and since $K$ is idempotent, we get $\sum_{g \in V^{\prime}}$ weight $\left(P_{u^{\prime}}^{\left(A_{g}\right)}\right)=$ weight $\left(P_{u}^{(A)}\right)$. On the other hand, for every $g \in V \backslash V^{\prime}$ and path $P_{u^{\prime}}^{\left(A_{g}\right)}$ of $A_{g}$, we have weight $\left(P_{u^{\prime}}^{\left(A_{g}\right)}\right)=0$. Therefore, we obtain

$$
\sum_{P_{u}^{(A)}} \text { weight }\left(P_{u}^{(A)}\right)=\sum_{g \in V} \sum_{P_{u^{\prime}}^{\left(A_{g}\right)}} \text { weight }\left(P_{u^{\prime}}^{\left(A_{g}\right)}\right) .
$$

We define the valid relabeling $h^{\prime} \in V R\left(\Gamma_{V}\right)$ as follows: 
- $h^{\prime}(z)=h(z)$ for every $z \in Z \backslash Z_{h}$,

and we let, nondeterministically,

- $h^{\prime}(z) \in \Sigma \backslash\left(\Sigma_{A} \cup \Sigma^{\prime} \cup h\left(Z \backslash Z_{h}\right) \cup\{w(i) \mid 0 \leq i \leq n-1\right.$ and $\left.w(i) \in h(y)\}\right)$ for every $z \in Z_{h}$.

Then we have $w \in h^{\prime}\left(u^{\prime}\right)$ which implies that $u^{\prime} \in \operatorname{preim}_{\Gamma_{V}}(w)$.

Conversely, let $u^{\prime}=u^{\prime}(0) \ldots u^{\prime}(n-1) \in \operatorname{preim}_{\Gamma_{V}}(w)$. Hence, there is a valid relabeling $h^{\prime} \in V R\left(\Gamma_{V}\right)$ such that $w \in h^{\prime}\left(u^{\prime}\right)$. By construction of $A_{\left(\Sigma^{\prime}, V\right)}$, there is a valid relabeling $h$ from $\Gamma_{A}$ to $\Sigma$ and a word $u=u(0) \ldots u(n-1) \in \Gamma_{A}^{*}$ such that

$$
u(i)= \begin{cases}u^{\prime}(i) & \text { if } u^{\prime}(i) \in \Sigma_{A} \cup Z \backslash Z_{h} \\ z & \text { if } u^{\prime}(i)=h(z) \text { and } z \in Z_{h} \\ y & \text { if } u^{\prime}(i) \in\left(h(y) \cap \Sigma^{\prime}\right) \cup\{y\}\end{cases}
$$

for every $0 \leq i \leq n-1$. Keeping the previous notations, for every $g \in V^{\prime}$, there is a path $P_{u^{\prime}}^{\left(A_{g}\right)}$ of the weighted automaton $A_{g}$ over $u^{\prime}$. By construction of $A_{\left(\Sigma^{\prime}, V\right)}$, all such paths $P_{u^{\prime}}^{\left(A_{g}\right)}\left(g \in V^{\prime}\right)$ have the same weight and there exist $r=\operatorname{card}\left(V^{\prime}\right)$ such paths. Furthermore, for every $g \in V^{\prime}$ and $P_{u^{\prime}}^{\left(A_{g}\right)}$ there is a path $P_{u}^{(A)}$ of $A$ over $u$ with weight $\left(P_{u}^{(A)}\right)=$ weight $\left(P_{u^{\prime}}^{\left(A_{g}\right)}\right)$, and since $K$ is idempotent we get weight $\left(P_{u}^{(A)}\right)=\sum_{g \in V^{\prime}}$ weight $\left(P_{u^{\prime}}^{\left(A_{g}\right)}\right)$. On the other hand, for every $g \in V \backslash V^{\prime}$ and path $P_{u^{\prime}}^{\left(A_{g}\right)}$ of $A_{g}$, we have that weight $\left(P_{u^{\prime}}^{\left(A_{g}\right)}\right)=0$. Therefore $\sum_{g \in V} \sum_{P_{u^{\prime}}^{\left(A_{g}\right)}}$ weight $\left(P_{u^{\prime}}^{\left(A_{g}\right)}\right)=\sum_{P_{u}^{(A)}}$ weight $\left(P_{u}^{(A)}\right)$. We consider the relabeling $h^{\prime \prime}$ from $\Gamma_{A}$ to $\Sigma$ defined in the following way. It is the identity on $\Sigma_{A}, h^{\prime \prime}(z)=$ $h^{\prime}(z)$ for every $z \in Z \backslash Z_{h}, h^{\prime \prime}(z)=h(z)$ for every $z \in Z_{h}$, and $h^{\prime \prime}(y)=h^{\prime}(y) \cup$ $\left(\left(h(y) \cap \Sigma^{\prime}\right) \backslash h\left(Z_{h}\right)\right)$ (in fact $\left(h(y) \cap \Sigma^{\prime}\right) \cap h\left(Z_{h}\right)=\emptyset$ since $h$ is a valid relabeling on $\left.\Gamma_{A}\right)$. Trivially $h^{\prime \prime}$ is a valid relabeling and $w \in h^{\prime \prime}(u)$ which implies that $u \in$ $\operatorname{preim}_{\Gamma_{A}}(w)$.

We conclude that for every $w \in \Sigma^{*}$ we have

$$
\begin{aligned}
\left(\left\|\mathcal{A}_{\left(\Sigma^{\prime}, V\right)}\right\|, w\right) & =\sum_{u^{\prime} \in \text { preim }_{\Gamma_{V}}(w)}\left(\left\|A_{\left(\Sigma^{\prime}, V\right)}\right\|, u^{\prime}\right)=\sum_{u^{\prime} \in \text { preim }_{\Gamma_{V}}(w)} \sum_{g \in V}\left(\left\|A_{g}\right\|, u^{\prime}\right) \\
& =\sum_{u^{\prime} \in \text { preim }_{\Gamma_{V}}(w)} \sum_{g \in V} \sum_{P_{u^{\prime}}^{\left(A_{g}\right)}} \text { weight }\left(P_{u^{\prime}}^{\left(A_{g}\right)}\right) \\
& =\sum_{u \in \operatorname{preim}_{\Gamma_{A}}(w)} \sum_{P_{u}^{(A)}} \text { weight }\left(P_{u}^{(A)}\right) \\
& =\sum_{u \in \text { preim }_{\Gamma_{A}}(w)}(\|A\|, u)=(\|\mathcal{A}\|, w)
\end{aligned}
$$

and we are done. 


\section{Closure properties of the class $V \operatorname{Rec}(K, \Sigma)$}

In this section, we investigate closure properties of the class of v-recognizable series over the infinite alphabet $\Sigma$ and the semiring $K$. More precisely, we show that the class $V \operatorname{Rec}(K, \Sigma)$ is closed under sum, and under scalar, Hadamard, Cauchy and shuffle products, as well as star operation.

Proposition 4. The class $\operatorname{VRec}(K, \Sigma)$ is closed under sum.

Proof. Let $r^{(i)} \in V \operatorname{Rec}(K, \Sigma)$ with $i=1,2$. Then there exist two wva $\mathcal{A}^{(i)}=$ $\left\langle\Sigma, A^{(i)}\right\rangle$ with $A^{(i)}=\left(Q^{(i)}, i n^{(i)}, w t^{(i)}, \operatorname{ter}^{(i)}\right)$ and $\Gamma^{(i)}=\Sigma^{(i)} \cup Z^{(i)} \cup\left\{y^{(i)}\right\}$, accepting $r^{(i)}$, for $i=1,2$. Without any loss, we assume that $Q^{(1)} \cap Q^{(2)}=\emptyset$ and $\left(Z^{(1)} \cup\left\{y^{(1)}\right\}\right) \cap\left(Z^{(2)} \cup\left\{y^{(2)}\right\}\right)=\emptyset$. We consider the wva $\mathcal{A}_{\left(\Sigma^{(2)}, V_{1}\right)}^{(1)}=$ $\left\langle\Sigma, A_{\left(\Sigma^{(2)}, V_{1}\right)}^{(1)}\right\rangle$ with $A_{\left(\Sigma^{(2)}, V_{1}\right)}^{(1)}=\left(Q_{V_{1}}^{(1)}, i n_{V_{1}}^{(1)}, w t_{V_{1}}^{(1)}, \operatorname{ter}_{V_{1}}^{(1)}\right)$ over $\Gamma^{(1)} \cup \Sigma^{(2)}$ and $K$ and the wva $\mathcal{A}_{\left(\Sigma^{(1)}, V_{2}\right)}^{(2)}=\left\langle\Sigma, A_{\left(\Sigma^{(1)}, V_{2}\right)}^{(2)}\right\rangle$ with $A_{\left(\Sigma^{(1)}, V_{2}\right)}^{(2)}=\left(Q_{V_{2}}^{(2)}, i n_{V_{2}}^{(2)}, w t_{V_{2}}^{(2)}, \operatorname{ter}_{V_{2}}^{(2)}\right)$ over $\Gamma^{(2)} \cup \Sigma^{(1)}$ and $K$, determined by the procedure before Lemma 1. Moreover, without any loss, we assume that $Q_{V_{1}}^{(1)} \cap Q_{V_{2}}^{(2)}=\emptyset$. By Lemma 1 we have $\left\|\mathcal{A}_{\left(\Sigma^{(2)}, V_{1}\right)}^{(1)}\right\|=r^{(1)}$ and $\left\|\mathcal{A}_{\left(\Sigma^{(1)}, V_{2}\right)}^{(2)}\right\|=r^{(2)}$. Let $Q=Q_{V_{1}}^{(1)} \cup Q_{V_{2}}^{(2)}$ and $\Gamma=$ $\Sigma^{(1)} \cup \Sigma^{(2)} \cup Z^{(1)} \cup Z^{(2)} \cup\{y\}$, where $y$ denotes a new free variable different from $y^{(1)}$ and $y^{(2)}$. We consider the wva $\mathcal{A}=\langle\Sigma, A\rangle$ with $A=(Q, i n, w t, t e r)$ where $i n$ and ter are defined, for every $q \in Q$, respectively by

$$
\operatorname{in}(q)=\left\{\begin{array}{ll}
i n_{V_{1}}^{(1)}(q) & \text { if } q \in Q_{V_{1}}^{(1)} \\
i n_{V_{2}}^{(2)}(q) & \text { if } q \in Q_{V_{2}}^{(2)}
\end{array} \quad \text { and } \quad \operatorname{ter}(q)=\left\{\begin{array}{ll}
\operatorname{ter}_{V_{1}}^{(1)}(q) & \text { if } q \in Q_{V_{1}}^{(1)} \\
\operatorname{ter}_{V_{2}}^{(2)}(q) & \text { if } q \in Q_{V_{2}}^{(2)}
\end{array} .\right.\right.
$$

The weight assignment mapping $w t: Q \times \Gamma \times Q \rightarrow K$ is defined as follows:

$$
w t\left(\left(q, \sigma, q^{\prime}\right)\right)= \begin{cases}w t_{V_{1}}^{(1)}\left(\left(q, \sigma, q^{\prime}\right)\right) & \text { if } q, q^{\prime} \in Q_{V_{1}}^{(1)}, \sigma \in \Gamma \backslash\{y\} \\ w t_{V_{2}}^{(2)}\left(\left(q, \sigma, q^{\prime}\right)\right) & \text { if } q, q^{\prime} \in Q_{V_{2}}^{(2)}, \sigma \in \Gamma \backslash\{y\} \\ w t_{V_{1}}^{(1)}\left(\left(q, y^{(1)}, q^{\prime}\right)\right) & \text { if } q, q^{\prime} \in Q_{V_{1}}^{(1)}, \sigma=y \\ w t_{V_{2}}^{(2)}\left(\left(q, y^{(2)}, q^{\prime}\right)\right) & \text { if } q, q^{\prime} \in Q_{V_{2}}^{(2)}, \sigma=y \\ 0 & \text { otherwise }\end{cases}
$$

for every $q, q^{\prime} \in Q, \sigma \in \Gamma$.

We show that $\|\mathcal{A}\|=\left\|\mathcal{A}^{(1)}\right\|+\left\|\mathcal{A}^{(2)}\right\|$. For this, let $w \in \Sigma^{*}, u \in \operatorname{preim}_{\Gamma}(w)$, and $h \in V R(\Gamma)$ such that $w \in h(u)$. Then, for every path $P_{u}^{(A)}$ of $A$ over $u$, by construction of $A$, we point out the following cases. (i) There exists a path $P_{u^{(1)}}$ of $A_{\left(\Sigma^{(2)}, V_{1}\right)}^{(1)}$ over $u^{(1)}$ with weight $\left(P_{u^{(1)}}\right)=$ weight $\left(P_{u}^{(A)}\right)$, where $u^{(1)}$ is obtained from $u$ by replacing every occurrence of $y$ with $y^{(1)}$. (ii) There exists a path $P_{u^{(2)}}$ of $A_{\left(\Sigma^{(1)}, V_{2}\right)}^{(2)}$ over $u^{(2)}$ with weight $\left(P_{u^{(2)}}\right)=$ weight $\left(P_{u}^{(A)}\right)$, where $u^{(2)}$ 
is obtained from $u$ by replacing every occurrence of $y$ with $y^{(2)}$. Suppose firstly that (i) holds. We consider the valid relabeling $h^{(1)} \in V R\left(\Gamma^{(1)} \cup \Sigma^{(2)}\right)$ such that $h^{(1)}$ coincides with $h$ on $\Sigma^{(1)} \cup \Sigma^{(2)} \cup Z^{(1)}$ and $h^{(1)}\left(y^{(1)}\right)=h(y) \cup h\left(Z^{(2)}\right)$. Trivially, $w \in h^{(1)}\left(u^{(1)}\right)$ which implies that $u^{(1)} \in \operatorname{preim}_{\Gamma^{(1)} \cup \Sigma^{(2)}}(w)$. Similarly, in case (ii) we get that $u^{(2)} \in \operatorname{preim}_{\Gamma^{(2)} \cup \Sigma^{(1)}}(w)$.

Conversely, let $w \in \Sigma^{*}, u^{(1)} \in \operatorname{preim}_{\Gamma^{(1)} \cup \Sigma^{(2)}}(w)$, and $h^{(1)} \in V R\left(\Gamma^{(1)} \cup \Sigma^{(2)}\right)$ such that $w \in h^{(1)}\left(u^{(1)}\right)$. Then, for every path $P_{u^{(1)}}$ of $A_{\left(\Sigma^{(2)}, V_{1}\right)}^{(1)}$ over $u^{(1)}$, by construction of $A$, there exists a path $P_{u}^{(A)}$ of $A$ over $u$ with weight $\left(P_{u}^{(A)}\right)=$ weight $\left(P_{u^{(1)}}\right)$, where $u$ is obtained from $u^{(1)}$ by replacing every occurrence of $y^{(1)}$ with $y$. We define the valid relabeling $h \in V R(\Gamma)$ which coincides with $h^{(1)}$ on $\Sigma^{(1)} \cup \Sigma^{(2)} \cup Z^{(1)}, h(z) \in \Sigma \backslash\left(\Sigma^{(1)} \cup \Sigma^{(2)} \cup h^{(1)}\left(Z^{(1)}\right) \cup\left(h^{(1)}\left(y^{(1)}\right)\right.\right.$

$\cap\{w(i) \mid 0 \leq i \leq n-1\}))$ for $z \in Z^{(2)}$ and $h(y)=h^{(1)}\left(y^{(1)}\right) \backslash\left\{h(z) \mid z \in Z^{(2)}\right\}$. Trivially, $w \in h(u)$ which implies that $u \in \operatorname{preim}_{\Gamma}(w)$.

Next assume that $u^{(2)} \in \operatorname{preim}_{\Gamma^{(2)} \cup \Sigma^{(1)}}(w)$ and $h^{(2)} \in V R\left(\Gamma^{(2)} \cup \Sigma^{(1)}\right)$ such that $w \in h^{(2)}\left(u^{(2)}\right)$. Then, for every path $P_{u^{(2)}}$ of $A_{\left(\Sigma^{(1)}, V_{2}\right)}^{(2)}$ over $u^{(2)}$, by construction of $A$, there exists a path $P_{u^{\prime}}^{A}$ of $A$ over $u^{\prime}$ with weight $\left(P_{u^{\prime}}^{(A)}\right)=$ weight $\left(P_{u^{(2)}}\right)$, where $u^{\prime}$ is obtained from $u^{(2)}$ by replacing every occurrence of $y^{(2)}$ with $y$. We define the valid relabeling $h^{\prime} \in V R(\Gamma)$ which coincides with $h^{(2)}$ on $\Sigma^{(1)} \cup \Sigma^{(2)} \cup Z^{(2)}$, $h^{\prime}(z) \in \Sigma \backslash\left(\Sigma^{(1)} \cup \Sigma^{(2)} \cup h^{(2)}\left(Z^{(2)}\right) \cup\left(h^{(2)}\left(y^{(2)}\right) \cap\{w(i) \mid 0 \leq i \leq n-1\}\right)\right)$ for $z \in Z^{(1)}$ and $h^{\prime}(y)=h^{(2)}\left(y^{(2)}\right) \backslash\left\{h^{\prime}(z) \mid z \in Z^{(1)}\right\}$. Trivially, $w \in h^{\prime}\left(u^{\prime}\right)$ which implies that $u^{\prime} \in \operatorname{preim}_{\Gamma}(w)$.

We conclude that for every $w \in \Sigma^{*}$ we have

$$
\begin{aligned}
& (\|\mathcal{A}\|, w)=\sum_{u \in \operatorname{preim}_{\Gamma}(w)}(\|A\|, u)=\sum_{u \in \operatorname{preim}_{\Gamma}(w)} \sum_{P_{u}^{(A)}} \text { weight }\left(P_{u}^{(A)}\right) \\
& =\sum_{u^{(1)} \in \text { preim }_{\Gamma^{(1)} \cup \Sigma^{(2)}}} \sum_{P_{u^{(1)}}} \text { weight }\left(P_{u^{(1)}}\right) \\
& +\sum_{u^{(2)} \in \operatorname{preim}_{\Gamma^{(2)} \cup \Sigma^{(1)}}} \sum_{P_{u^{(2)}}} \text { weight }\left(P_{u^{(2)}}\right) \\
& =\sum_{u^{(1)} \in \operatorname{preim}_{\Gamma^{(1)} \cup \Sigma^{(2)}}(w)}\left(\left\|A_{\left(\Sigma^{(2)}, V_{1}\right)}^{(1)}\right\|, u^{(1)}\right) \\
& +\sum_{u^{(2)} \in \text { preim }_{\Gamma^{(2)} \cup \Sigma^{(1)}}(w)}\left(\left\|A_{\left(\Sigma^{(1)}, V_{2}\right)}^{(2)}\right\|, u^{(2)}\right) \\
& =\left(\left\|\mathcal{A}_{\left(\Sigma^{(2)}, V_{1}\right)}^{(1)}\right\|, w\right)+\left(\left\|\mathcal{A}_{\left(\Sigma^{(1)}, V_{2}\right)}^{(2)}\right\|, w\right) \\
& =\left(\left\|\mathcal{A}^{(1)}\right\|, w\right)+\left(\left\|\mathcal{A}^{(2)}\right\|, w\right) \\
& =\left(\left\|\mathcal{A}^{(1)}\right\|+\left\|\mathcal{A}^{(2)}\right\|, w\right)=\left(r^{(1)}+r^{(2)}, w\right)
\end{aligned}
$$


where the sixth equality holds by Lemma 1, and we are done.

Proposition 5. The class $V \operatorname{Rec}(K, \Sigma)$ is closed under the scalar products.

Proof. Let $r \in V \operatorname{Rec}(K, \Sigma)$ and $k \in K$. Then there exists a wva $\mathcal{A}=\langle\Sigma, A\rangle$ with $A=(Q, i n, w t, t e r)$ accepting $r$. We consider the wva $\mathcal{A}^{\prime}=\left\langle\Sigma, A^{\prime}\right\rangle$ with $A^{\prime}=\left(Q, i n^{\prime}, w t, t e r\right)$ where $i n^{\prime}(q)=k \cdot i n(q)$ for every $q \in Q$. Then, by standard arguments we get $\left\|\mathcal{A}^{\prime}\right\|=k\|\mathcal{A}\|$, and we are done.

Proposition 6. The class VRec $(K, \Sigma)$ is closed under Hadamard product.

Proof. Let $r^{(i)} \in V \operatorname{Rec}(K, \Sigma)$ with $i=1,2$. Then there exist two wva $\mathcal{A}^{(i)}=$ $\left\langle\Sigma, A^{(i)}\right\rangle$ with $A^{(i)}=\left(Q^{(i)}, i n^{(i)}, w t^{(i)}\right.$, ter $\left.^{(i)}\right)$ over $\Gamma^{(i)}=\Sigma^{(i)} \cup Z^{(i)} \cup\left\{y^{(i)}\right\}$, accepting $r^{(i)}$ for $i=1,2$. Without any loss, we assume that $Q^{(1)} \cap Q^{(2)}=\emptyset$ and $\left(Z^{(1)} \cup\left\{y^{(1)}\right\}\right) \cap\left(Z^{(2)} \cup\left\{y^{(2)}\right\}\right)=\emptyset$. We consider the wva $\mathcal{A}_{\left(\Sigma^{(2)}, V_{1}\right)}^{(1)}=$ $\left\langle\Sigma, A_{\left(\Sigma^{(2)}, V_{1}\right)}^{(1)}\right\rangle$ with $A_{\left(\Sigma^{(2)}, V_{1}\right)}^{(1)}=\left(Q_{V_{1}}^{(1)}, i n_{V_{1}}^{(1)}, w t_{V_{1}}^{(1)}, \operatorname{ter}_{V_{1}}^{(1)}\right)$ over $\Gamma^{(1)} \cup \Sigma^{(2)}$ and $\mathcal{A}_{\left(\Sigma^{(1)}, V_{2}\right)}^{(2)}=\left\langle\Sigma, A_{\left(\Sigma^{(1)}, V_{2}\right)}^{(2)}\right\rangle$ with $A_{\left(\Sigma^{(1)}, V_{2}\right)}^{(2)}=\left(Q_{V_{2}}^{(2)}, i n_{V_{2}}^{(2)}, w t_{V_{2}}^{(2)}, \operatorname{ter}_{V_{2}}^{(2)}\right)$ over $\Gamma^{(2)} \cup$ $\Sigma^{(1)}$ determined by the procedure described before Lemma 1. Moreover, without any loss, we assume that $Q_{V_{1}}^{(1)} \cap Q_{V_{2}}^{(2)}=\emptyset$. By Lemma 1 we get $\left\|\mathcal{A}_{\left(\Sigma^{(2)}, V_{1}\right)}^{(1)}\right\|=r^{(1)}$ and $\left\|\mathcal{A}_{\left(\Sigma^{(1)}, V_{2}\right)}^{(2)}\right\|=r^{(2)}$.

We consider the set $\left(Z^{(1)} \cup\left\{y^{(1)}\right\}\right) \times\left(Z^{(2)} \cup\left\{y^{(2)}\right\}\right) \backslash\{y\}$ where $y=\left(y^{(1)}, y^{(2)}\right)$, and a maximal subset $G \subseteq\left(Z^{(1)} \cup\left\{y^{(1)}\right\}\right) \times\left(Z^{(2)} \cup\left\{y^{(2)}\right\}\right) \backslash\{y\}$ satisfying the next condition: every element of $Z^{(1)}$ (resp. of $Z^{(2)}$ ) occurs in at most one pair in $G$ as a left (resp. as a right) coordinate. Assume that $G_{1}, \ldots, G_{m}$ is an enumeration of all such sets of pairs of variables. Moreover, we let $Q=Q_{V_{1}}^{(1)} \times Q_{V_{2}}^{(2)}$ and $\Gamma_{G_{j}}=$ $\Sigma^{(1)} \cup \Sigma^{(2)} \cup G_{j} \cup\{y\}$ for every $1 \leq j \leq m$, and we consider the wva $\mathcal{A}_{G_{j}}=$ $\left\langle\Sigma, A_{G_{j}}\right\rangle$ with $A_{G_{j}}=\left(Q, i n_{G_{j}}, w t_{G_{j}}\right.$, ter $\left._{G_{j}}\right)$ over $\Gamma_{G_{j}}$. For every $1 \leq j \leq m$, the initial and terminal distribution are given respectively, by $i_{G_{j}}\left(\left(q^{(1)}, q^{(2)}\right)\right)=$ $i n_{V_{1}}^{(1)}\left(q^{(1)}\right) \cdot i n_{V_{2}}^{(2)}\left(q^{(2)}\right)$ and $\operatorname{ter}_{G_{j}}\left(\left(q^{(1)}, q^{(2)}\right)\right)=\operatorname{ter}_{V_{1}}^{(1)}\left(q^{(1)}\right) \cdot \operatorname{ter}_{V_{2}}^{(2)}\left(q^{(2)}\right)$, and the weight assignment mapping $w_{G_{j}}: Q \times \Gamma_{G_{j}} \times Q \rightarrow K$ is defined by

$$
\begin{gathered}
w t_{G_{j}}\left(\left(q^{(1)}, q^{(2)}\right), \sigma,\left(q^{(1)}, q^{\prime(2)}\right)\right)= \\
\begin{cases}w t_{V_{1}}^{(1)}\left(\left(q^{(1)}, \sigma, q^{(1)}\right)\right) \cdot w t_{V_{2}}^{(2)}\left(\left(q^{(2)}, \sigma, q^{(2)}\right)\right) & \text { if } \sigma \in \Sigma^{(1)} \cup \Sigma^{(2)} \\
w t_{V_{1}}^{(1)}\left(\left(q^{(1)}, x^{(1)}, q^{\prime(1)}\right)\right) \cdot w t_{V_{2}}^{(2)}\left(\left(q^{(2)}, x^{(2)}, q^{(2)}\right)\right) & \text { if } \sigma=\left(x^{(1)}, x^{(2)}\right) \in G_{j} \cup\{y\} \\
0 & \text { otherwise }\end{cases}
\end{gathered}
$$

for every $\left(q^{(1)}, q^{(2)}\right),\left(q^{\prime(1)}, q^{(2)}\right) \in Q, \sigma \in \Gamma_{G_{j}}$. 
By Proposition 4 , the series $\sum_{1 \leq j \leq m}\left\|\mathcal{A}_{G_{j}}\right\|$ is recognizable. We will show that $\left\|\mathcal{A}^{(1)}\right\| \odot\left\|\mathcal{A}^{(2)}\right\|=\sum_{1 \leq j \leq m}\left\|\mathcal{A}_{G_{j}}\right\|$.

To this end, let $w=w(0) \ldots w(n-1) \in \Sigma^{*}, u^{(1)} \in \operatorname{preim}_{\Gamma^{(1)} \cup \Sigma^{(2)}}(w), u^{(2)} \in$ $\operatorname{preim}_{\Gamma^{(2)} \cup \Sigma^{(1)}}(w), h^{(1)} \in V R\left(\Gamma^{(1)} \cup \Sigma^{(2)}\right)$, and $h^{(2)} \in V R\left(\Gamma^{(2)} \cup \Sigma^{(1)}\right)$ such that $w \in h^{(1)}\left(u^{(1)}\right) \cap h^{(2)}\left(u^{(2)}\right)$. For every $w(t) \in \Sigma, 0 \leq t \leq n-1$, we have either $w(t) \in \Sigma^{(1)} \cup \Sigma^{(2)}$ and hence $u^{(1)}(t)=u^{(2)}(t)=w(t)$, or $w(t) \in \Sigma \backslash \Sigma^{(1)} \cup \Sigma^{(2)}$ and one of the following cases holds.

- There exist bounded variables $z^{(1)} \in Z^{(1)}, z^{(2)} \in Z^{(2)}$ such that $u^{(1)}(t)=z^{(1)}$, $u^{(2)}(t)=z^{(2)}$ and $h^{(1)}\left(u^{(1)}(t)\right)=h^{(2)}\left(u^{(2)}(t)\right)=w(t)$.

- There exists a bounded variable $z^{(1)} \in Z^{(1)}$ such that $u^{(1)}(t)=z^{(1)}, u^{(2)}(t)=$ $y^{(2)}$ and $h^{(1)}\left(u^{(1)}(t)\right)=w(t) \in h^{(2)}\left(u^{(2)}(t)\right)$.

- There exists a bounded variable $z^{(2)} \in Z^{(2)}$ such that $u^{(1)}(t)=y^{(1)}, u^{(2)}(t)=$ $z^{(2)}$ and $h^{(2)}\left(u^{(2)}(t)\right)=w(t) \in h^{(1)}\left(u^{(1)}(t)\right)$.

- $u^{(1)}(t)=y^{(1)}, u^{(2)}(t)=y^{(2)}$, and $w(t) \in h^{(1)}\left(u^{(1)}(t)\right) \cap h^{(2)}\left(u^{(2)}(t)\right)$.

We consider the word $u=u(0) \ldots u(n-1)$ by

$$
u(t)= \begin{cases}w(t) & \text { if } w(t) \in \Sigma^{(1)} \cup \Sigma^{(2)} \\ \left(u^{(1)}(t), u^{(2)}(t)\right) & \text { otherwise }\end{cases}
$$

for every $0 \leq t \leq n-1$. For every $1 \leq j \leq m$, we define a valid relabeling $h_{j} \in V R\left(\Gamma_{G_{j}}\right)$ such that $h_{j}(\sigma)=h^{(1)}\left(x^{(1)}\right)$ for every $\sigma=\left(x^{(1)}, x^{(2)}\right) \in$ $\left(Z^{(1)} \times\left(Z^{(2)} \cup\left\{y^{(2)}\right\}\right)\right) \cap G_{j}$, and $h_{j}(\sigma)=h^{(2)}\left(x^{(2)}\right)$ for every $\sigma=\left(x^{(1)}, x^{(2)}\right) \in$ $\left(\left\{y^{(1)}\right\} \times Z^{(2)}\right) \cap G_{j}$. Hence $u \in \operatorname{preim}_{\Gamma_{G_{j}}}(w)$ for some $1 \leq j \leq m$.

By the definition of the list $G_{1}, \ldots, G_{m}$, there is a set $J \subseteq\{1, \ldots, m\}$, such that for every path

$$
P_{u^{(1)}}:\left(q_{0}^{(1)}, u^{(1)}(0), q_{1}^{(1)}\right) \ldots\left(q_{n-1}^{(1)}, u^{(1)}(n-1), q_{n}^{(1)}\right)
$$

of $A_{\left(\Sigma^{(2)}, V_{1}\right)}^{(1)}$ over $u^{(1)}$, and

$$
P_{u^{(2)}}:\left(q_{0}^{(2)}, u^{(2)}(0), q_{1}^{(2)}\right) \ldots\left(q_{n-1}^{(2)}, u^{(2)}(n-1), q_{n}^{(2)}\right)
$$

of $A_{\left(\Sigma^{(1)}, V_{2}\right)}^{(2)}$ over $u^{(2)}$, there exists a path

$$
P_{u}^{\left(G_{j}\right)}:\left(\left(q_{0}^{(1)}, q_{0}^{(2)}\right), u(0),\left(q_{1}^{(1)}, q_{1}^{(2)}\right)\right) \ldots\left(\left(q_{n-1}^{(1)}, q_{n-1}^{(2)}\right), u(n-1),\left(q_{n}^{(1)}, q_{n}^{(2)}\right)\right)
$$

of $A_{G_{j}}$ over $u$, for every $j \in J$. Conversely, for every path $P_{u}^{\left(G_{j}\right)}$ of $A_{G_{j}}$ over $u(j \in$ $J)$ there are two paths $P_{u^{(1)}}$ of $A_{\left(\Sigma^{(2)}, V_{1}\right)}^{(1)}$ over $u^{(1)}$ and $P_{u^{(2)}}$ of $A_{\left(\Sigma^{(1)}, V_{2}\right)}^{(2)}$ over $u^{(2)}$ respectively, obtained in the obvious way. Moreover, in case weight $\left(P_{u}^{\left(G_{j}\right)}\right) \neq 0$, for every $j \in J$, it holds 


$$
\begin{aligned}
& \text { weight }\left(P_{u}^{\left(G_{j}\right)}\right) \\
& \begin{array}{c}
=i n_{G_{j}}\left(\left(q_{0}^{(1)}, q_{0}^{(2)}\right)\right) \cdot \prod_{0 \leq t \leq n-1} w t_{G_{j}}\left(\left(\left(q_{t}^{(1)}, q_{t}^{(2)}\right), u(t),\left(q_{t+1}^{(1)}, q_{t+1}^{(2)}\right)\right)\right) \\
\cdot \operatorname{ter}_{G_{j}}\left(\left(q_{n}^{(1)}, q_{n}^{(2)}\right)\right) \\
=i n_{V_{1}}^{(1)}\left(q_{0}^{(1)}\right) \cdot i n_{V_{2}}^{(2)}\left(q_{0}^{(2)}\right) \cdot \prod_{0 \leq t \leq n-1}\left(\begin{array}{c}
w t_{V_{1}}^{(1)}\left(\left(q_{t}^{(1)}, u^{(1)}(t), q_{t+1}^{(1)}\right)\right) \\
\cdot w t_{V_{2}}^{(2)}\left(\left(q_{t}^{(2)}, u^{(2)}(t), q_{t+1}^{(2)}\right)\right)
\end{array}\right) \\
=i n_{V_{1}}^{(1)}\left(q_{0}^{(1)}\right) \cdot \prod_{0 \leq t \leq n-1} w t_{V_{1}}^{(1)}\left(\left(q_{t}^{(1)}, u^{(1)}(t), q_{t+1}^{(1)}\right)\right) \cdot \operatorname{ter}_{V_{1}}^{(1)}\left(q_{n}^{(1)}\right) \\
\cdot i n_{V_{2}}^{(2)}\left(q_{0}^{(2)}\right) \cdot \prod_{0 \leq t \leq n-1}^{(2)} w t_{V_{2}}^{(2)}\left(\left(q_{t}^{(2)}, u^{(2)}(t), q_{t+1}^{(2)}\right)\right) \cdot \operatorname{ter}_{V_{2}}^{(2)}\left(q_{n}^{(2)}\right)
\end{array}
\end{aligned}
$$

$=\operatorname{weight}\left(P_{u^{(1)}}\right) \cdot \operatorname{weight}\left(P_{u^{(2)}}\right)$.

Conversely, if weight $\left(P_{u^{(1)}}\right) \neq 0$, weight $\left(P_{u^{(2)}}\right) \neq 0$, then by the consideration of the list $G_{1}, \ldots, G_{m}$, there is at least one $1 \leq j \leq m$ with weight $\left(P_{u}^{\left(G_{j}\right)}\right)=$ weight $\left(P_{u^{(1)}}\right) \cdot$ weight $\left(P_{u^{(2)}}\right)$. Therefore, and since $K$ is idempotent, we obtain ${ }^{2}$

$$
\sum_{1 \leq j \leq m} \sum_{P_{u}^{\left(G_{j}\right)}} \text { weight }\left(P_{u}^{\left(G_{j}\right)}\right)=\sum_{P_{u^{(1)}}, P_{u}(2)} \text { weight }\left(P_{u^{(1)}}\right) \cdot \text { weight }\left(P_{u^{(2)}}\right) \text {. }
$$

We conclude

$$
\begin{aligned}
\left(\sum_{1 \leq j \leq m}\left\|\mathcal{A}_{G_{j}}\right\|, w\right) & =\sum_{1 \leq j \leq m}\left(\left\|\mathcal{A}_{G_{j}}\right\|, w\right)=\sum_{1 \leq j \leq m} \sum_{u \in \text { preim }_{\Gamma_{G_{j}}}(w)}\left(\left\|A_{G_{j}}\right\|, u\right) \\
& =\sum_{1 \leq j \leq m} \sum_{u \in \operatorname{preim}_{\Gamma_{G_{j}}}(w)} \sum_{P_{u}^{\left(G_{j}\right)}} \text { weight }\left(P_{u}^{\left(G_{j}\right)}\right) \\
& =\sum_{u^{(1)} \in \operatorname{preim}_{\Gamma^{(1)} \cup \Sigma^{(2)}}(w)} \sum_{P_{u(1)}}\left(\text { weight }\left(P_{u^{(1)}}\right) \cdot \text { weight }\left(P_{u^{(2)}}\right)\right) \\
& =\sum_{P_{u^{(2)}}} \sum_{u^{(1)} \in \operatorname{preim}_{\Gamma^{(1)} \cup \Sigma^{(2)}}(w)} \sum_{P_{u^{(1)}}} \text { weight }\left(P_{u^{(1)}}\right)
\end{aligned}
$$

\footnotetext{
${ }^{2}$ It should be clear that for $j \in\{1, \ldots, m\} \backslash J$ the paths $P_{u}^{(G j)}$ do not exist, hence by definition weight $\left(P_{u}^{(G j)}\right)=0$.
} 


$$
\begin{array}{r}
\sum_{u^{(2)} \in \text { preim }_{\Gamma^{(2)} \cup \Sigma^{(1)}}} \sum_{u^{(1)} \in \text { preim }_{\Gamma^{(1)} \cup \Sigma^{(2)}(w)}}\left(\left\|A_{\left.\Sigma^{(2)}, V_{1}\right)}^{(1)}\right\|, u^{(1)}\right) \\
\cdot \sum_{u^{(2)} \in \text { preim }_{\Gamma^{(2)} \cup \Sigma^{(1)}(w)}}\left(\left\|A_{\left(\Sigma^{(1)}, V_{2}\right)}^{(2)}\right\|, u^{(2)}\right) \\
=\left(\left\|\mathcal{A}_{\left(\Sigma^{(2)}, V_{1}\right)}^{(1)}\right\|, w\right) \cdot\left(\left\|\mathcal{A}_{\left(\Sigma^{(1)}, V_{2}\right)}^{(2)}\right\|, w\right)
\end{array}
$$

for every $w \in \Sigma^{*}$, which, by Lemma 1 , implies

$$
\left(\left\|\mathcal{A}^{(1)}\right\| \odot\left\|\mathcal{A}^{(2)}\right\|, w\right)=\left(\sum_{1 \leq j \leq m}\left\|\mathcal{A}_{G_{j}}\right\|, w\right)
$$

for every $w \in \Sigma^{*}$, i.e., $\left\|\mathcal{A}^{(1)}\right\| \odot\left\|\mathcal{A}^{(2)}\right\|=\sum_{1 \leq j \leq m}\left\|\mathcal{A}_{G_{j}}\right\|$, as required.

Proposition 7. The class VRec $(K, \Sigma)$ is closed under Cauchy product.

Proof. Let $r^{(i)} \in V \operatorname{Rec}(K, \Sigma)$ with $i=1,2$. We consider the proper series $r^{\prime(1)}, r^{\prime(2)}$ over $\Sigma$ and $K$ defined, for every $w \in \Sigma^{*}$, by

$$
\begin{aligned}
& \text { - }\left(r^{\prime(1)}, w\right)=\left\{\begin{array}{ll}
\left(r^{(1)}, w\right) & \text { if } w \in \Sigma^{+} \\
0 & \text { otherwise }
\end{array},\right. \text { and } \\
& \text { - }\left(r^{\prime(2)}, w\right)= \begin{cases}\left(r^{(2)}, w\right) & \text { if } w \in \Sigma^{+} \\
0 & \text { otherwise. }\end{cases}
\end{aligned}
$$

Then $r^{(1)} \cdot r^{(2)}=r^{\prime(1)} \cdot r^{\prime(2)}+\left(r^{(1)}, \varepsilon\right) r^{(2)}+r^{(1)}\left(r^{(2)}, \varepsilon\right)+\left(r^{(1)}, \varepsilon\right)\left(r^{(2)}, \varepsilon\right) \bar{\varepsilon}$ and by Propositions 2,4 , and 5 , it suffices to show that $r^{\prime(1)} \cdot r^{\prime(2)} \in V \operatorname{Rec}(K, \Sigma)$. By Proposition 3 , there are normalized wva $\mathcal{A}^{(i)}=\left\langle\Sigma, A^{(i)}\right\rangle$ with $A^{(i)}=\left(Q^{(i)}, q_{\text {in }}^{(i)}, w t^{(i)}, q_{\text {ter }}^{(i)}\right)$ over $\Gamma^{(i)}=\Sigma^{(i)} \cup Z^{(i)} \cup\left\{y^{(i)}\right\}$ and $K$, accepting respectively $r^{(i)}$, with $i=$ 1,2. Without any loss, we assume that $Q^{(1)} \cap Q^{(2)}=\emptyset$ and $\left(Z^{(1)} \cup\left\{y^{(1)}\right\}\right) \cap$ $\left(Z^{(2)} \cup\left\{y^{(2)}\right\}\right)=\emptyset$. We consider the wva $\mathcal{A}_{\left(\Sigma^{(2)}, V_{1}\right)}^{(1)}=\left\langle\Sigma, A_{\left(\Sigma^{(2)}, V_{1}\right)}^{(1)}\right\rangle$ and $\mathcal{A}_{\left(\Sigma^{(1)}, V_{2}\right)}^{(2)}=\left\langle\Sigma, A_{\left(\Sigma^{(1)}, V_{2}\right)}^{(2)}\right\rangle$ determined by the procedure before Lemma 1. By Proposition 3 and Lemma $1, \mathcal{A}_{\left(\Sigma^{(2)}, V_{1}\right)}^{(1)}$ and $\mathcal{A}_{\left(\Sigma^{(1)}, V_{2}\right)}^{(2)}$ can be assumed to be normalized hence, let $A_{\left(\Sigma^{(2)}, V_{1}\right)}^{(1)}=\left(Q_{V_{1}}^{(1)}, q_{i_{V_{1}}}^{(1)}, w t_{V_{1}}^{(1)}, q_{t e r_{V_{1}}}^{(1)}\right)$ over $\Gamma^{(1)} \cup \Sigma^{(2)}$ and $A_{\left(\Sigma^{(1)}, V_{2}\right)}^{(2)}=\left(Q_{V_{2}}^{(2)}, q_{i n_{V_{2}}}^{(2)}, w t_{V_{2}}^{(2)}, q_{t e r_{V_{2}}}^{(2)}\right)$ over $\Gamma^{(2)} \cup \Sigma^{(1)}$. Moreover, without any loss, 
we assume that $Q_{V_{1}}^{(1)} \cap Q_{V_{2}}^{(2)}=\emptyset$. We let $y=\left(y^{(1)}, y^{(2)}\right)$ and consider the set $H=$ $\left(Z^{(1)} \cup\left\{y^{(1)}\right\}\right) \times\left(Z^{(2)} \cup\left\{y^{(2)}\right\}\right) \backslash\{y\}$, and a maximal subset $G \subseteq H \cup Z^{(1)} \cup Z^{(2)}$ satisfying the following condition: every element of $Z^{(1)}$ (resp. of $Z^{(2)}$ ) occurs either in at most one pair of $H$ as a left (resp. as a right) coordinate, or as a single element of $G$. Assume that $G_{1}, \ldots, G_{m}$ is an enumeration of all such sets. We let $Q=Q_{V_{1}}^{(1)} \cup Q_{V_{2}}^{(2)} \backslash\left\{q_{t_{e V_{1}}}^{(1)}\right\}$ and consider, for every $1 \leq j \leq m$, the normalized wva $\mathcal{A}_{G_{j}}=\left\langle\Sigma, A_{G_{j}}\right\rangle$ where $A_{G_{j}}=\left(Q, q_{i n_{V_{1}}}^{(1)}, w t_{G_{j}}, q_{t e r_{V_{2}}}^{(2)}\right)$ and $\Gamma_{G_{j}}=$ $\Sigma^{(1)} \cup \Sigma^{(2)} \cup G_{j} \cup\{y\}$. The weight assignment mapping $w t_{G_{j}}$ is defined, for every $1 \leq j \leq m$, as follows:

$$
\begin{aligned}
& w t_{G_{j}}\left(\left(q, \sigma, q^{\prime}\right)\right)= \\
& \left\{\begin{array}{cc}
w t_{V_{1}}^{(1)}\left(\left(q, \sigma, q^{\prime}\right)\right) & \text { if } q, q^{\prime} \in Q_{V_{1}}^{(1)} \backslash\left\{q_{\text {ter }_{V_{1}}}^{(1)}\right\} \text { and } \\
\sigma \in \Sigma^{(1)} \cup \Sigma^{(2)} \cup\left(Z^{(1)} \cap G_{j}\right) \\
w t_{V_{2}}^{(2)}\left(\left(q, \sigma, q^{\prime}\right)\right) & \text { if } q, q^{\prime} \in Q_{V_{2}}^{(2)} \text { and } \sigma \in \Sigma^{(1)} \cup \Sigma^{(2)} \cup\left(Z^{(2)} \cap G_{j}\right) \\
w t_{V_{1}}^{(1)}\left(\left(q, \sigma, q_{\text {ter }_{V_{1}}}^{(1)}\right)\right) & \text { if } q \in Q_{V_{1}}^{(1)} \backslash\left\{q_{\text {ter }_{V_{1}}}^{(1)}\right\}, q^{\prime}=q_{\text {in }_{V_{2}}}^{(2)}, \text { and } \\
\sigma \in \Sigma^{(1)} \cup \Sigma^{(2)} \cup\left(Z^{(1)} \cap G_{j}\right) \\
w t_{V_{1}}^{(1)}\left(\left(q, x^{(1)}, q^{\prime}\right)\right) & \text { if } q, q^{\prime} \in Q_{V_{1}}^{(1)} \backslash\left\{q_{\text {ter }_{V_{1}}}^{(1)}\right\} \text { and } \\
w t_{V_{2}}^{(2)}\left(\left(q, x^{(2)}, q^{\prime}\right)\right) & \text { if } q, q^{\prime} \in Q_{V_{2}}^{(2)} \text { and } \sigma=\left(x^{(1)}, x^{(2)}\right) \in G_{j} \cup\{y\} \\
w t_{V_{1}}^{(1)}\left(\left(q, x^{(1)}, q_{\text {ter }_{V_{1}}}^{(1)}\right)\right) & \text { if } q \in Q_{V_{1}}^{(1)} \backslash\left\{q_{\text {ter }_{V_{1}}}^{(1)}\right\}, q^{\prime}=q_{\text {in }_{V_{2}}}^{(2)}, \text { and } \\
& \sigma=\left(x^{(1)}, x^{(2)}\right) \in G_{j} \cup\{y\} \\
0 & \text { otherwise }
\end{array}\right.
\end{aligned}
$$

for every $q, q^{\prime} \in Q, \sigma \in \Gamma_{G_{j}}$.

By Lemma 1 and Proposition 4 , it suffices to show that $\left\|\mathcal{A}_{\left(\Sigma^{(2)}, V_{1}\right)}^{(1)}\right\| \cdot\left\|\mathcal{A}_{\left(\Sigma^{(1)}, V_{2}\right)}^{(2)}\right\|=$ $\sum_{1 \leq j \leq m}\left\|\mathcal{A}_{G_{j}}\right\|$

Let $w_{1}, w_{2} \in \Sigma^{+}, u_{1} \in \operatorname{preim}_{\Gamma^{(1)} \cup \Sigma^{(2)}}\left(w_{1}\right)$, and $u_{2} \in \operatorname{preim}_{\Gamma^{(2)} \cup \Sigma^{(1)}}\left(w_{2}\right)$. We set $w_{1} w_{2}=w=w(0) \ldots w(n-1)$ hence, $w_{1}=w(0) \ldots w(k)$ and $w_{2}=w(k+$ 1) $\ldots w(n-1)$ for some $0 \leq k<n-1$. Furthermore, we set $u_{1} u_{2}=u=u(0) \ldots u(n-$ 1 ) and, by our assumption, we have $u_{1}=u(0) \ldots u(k)$ and $u_{2}=u(k+1) \ldots u(n-1)$. Let $h^{(1)} \in V R\left(\Gamma^{(1)} \cup \Sigma^{(2)}\right), h^{(2)} \in V R\left(\Gamma^{(2)} \cup \Sigma^{(1)}\right)$ such that $w_{1} \in h^{(1)}\left(u_{1}\right)$ and $w_{2} \in h^{(2)}\left(u_{2}\right)$. Moreover, let

$$
P_{u_{1}}:\left(q_{i n_{V_{1}}}^{(1)}, u(0), q_{1}^{(1)}\right) \ldots\left(q_{k-1}^{(1)}, u(k), q_{t e r_{V_{1}}}^{(1)}\right)
$$


be a path of $A_{\left(\Sigma^{(2)}, V_{1}\right)}^{(1)}$ over $u_{1}$, and

$$
P_{u_{2}}:\left(q_{\text {in }_{V_{2}}}^{(2)}, u(k+1), q_{k+1}^{(2)}\right) \ldots\left(q_{n-1}^{(2)}, u(n-1), q_{\text {ter }_{V_{2}}}^{(2)}\right)
$$

be a path of $A_{\left(\Sigma^{(1)}, V_{2}\right)}^{(2)}$ over $u_{2}$.

We point out the following cases.

- The sets $\{w(0), \ldots, w(k)\} \cap\left(\Sigma \backslash\left(\Sigma^{(1)} \cup \Sigma^{(2)}\right)\right)$ and $\{w(k+1), \ldots, w(n-1)\} \cap$ $\left(\Sigma \backslash\left(\Sigma^{(1)} \cup \Sigma^{(2)}\right)\right)$ are disjoint. Then, if weight $\left(P_{u_{1}}\right) \neq 0 \neq \operatorname{weight}\left(P_{u_{2}}\right)$, by the definition of the list $G_{1}, \ldots, G_{m}$, there is a set $J \subseteq\{1, \ldots, m\}$ such that for every $j \in J$

$$
\begin{array}{r}
P_{u^{\prime}}^{\left(G_{j}\right)}:\left(q_{i n_{V_{1}}}^{(1)}, u^{\prime}(0), q_{1}^{(1)}\right) \ldots\left(q_{k-1}^{(1)}, u^{\prime}(k), q_{i n_{V_{2}}}^{(2)}\right)\left(q_{i n_{V_{2}}}^{(2)}, u^{\prime}(k+1), q_{k+1}^{(2)}\right) \\
\ldots\left(q_{n-1}^{(2)}, u^{\prime}(n-1), q_{t e r_{V_{2}}}^{(2)}\right)
\end{array}
$$

is a path of $A_{G_{j}}$ over $u^{\prime}$, where $u^{\prime}$ is obtained by $u$ by replacing every occurrence of $y^{(1)}$ in $u_{1}$ and $y^{(2)}$ in $u_{2}$, respectively by $y$. Clearly, it holds weight $\left(P_{u^{\prime}}^{\left(G_{j}\right)}\right)=$ weight $\left(P_{u_{1}}\right)$ weight $\left(P_{u_{2}}\right)$. Moreover, since $K$ is idempotent, we get $\sum_{j \in J}$ weight $\left(P_{u^{\prime}}^{\left(G_{j}\right)}\right)=$ weight $\left(P_{u_{1}}\right)$ weight $\left(P_{u_{2}}\right)$. By the definition of the list $G_{1}, \ldots, G_{m}$ we have also weight $\left(P_{u^{\prime}}^{\left(G_{j}\right)}\right)=0$ for every $j \in$ $\{1, \ldots, m\} \backslash J$, and thus $\sum_{1 \leq j \leq m}$ weight $\left(P_{u^{\prime}}^{\left(G_{j}\right)}\right)=$ weight $\left(P_{u_{1}}\right)$ weight $\left(P_{u_{2}}\right)$. Furthermore, the relabeling $h^{(j)}: \Gamma_{G_{j}} \rightarrow \mathcal{P}(\Sigma)$, for every $1 \leq j \leq m$, which is defined as the identity on $\Sigma^{(1)} \cup \Sigma^{(2)}$, and

$-h^{(j)}\left(z^{(1)}\right)=h^{(1)}\left(z^{(1)}\right)$ for every $z^{(1)} \in Z^{(1)} \cap G_{j}$,

$-h^{(j)}\left(z^{(2)}\right)=h^{(2)}\left(z^{(2)}\right)$ for every $z^{(2)} \in Z^{(2)} \cap G_{j}$, and

- $h^{(j)}\left(\left(x^{(1)}, x^{(2)}\right)\right)$ are defined nondeterministically in $\Sigma \backslash\left(\Sigma^{(1)} \cup \Sigma^{(2)} \cup\right.$ $\left.\{w(0), \ldots, w(n-1)\} \cup\left\{h^{(j)}\left(z^{(i)}\right) \mid z^{(i)} \in Z^{(i)} \cap G_{j}, i=1,2\right\}\right)$ whenever $\left(x^{(1)}, x^{(2)}\right) \in G_{j} \backslash\{y\}$

is valid, and clearly $w \in h^{(j)}\left(u^{\prime}\right)$ for every $j \in J$.

- The sets $\{w(0), \ldots, w(k)\} \cap\left(\Sigma \backslash\left(\Sigma^{(1)} \cup \Sigma^{(2)}\right)\right)$ and $\{w(k+1), \ldots, w(n-1)\}$ $\cap\left(\Sigma \backslash\left(\Sigma^{(1)} \cup \Sigma^{(2)}\right)\right)$ are not disjoint. For simplicity, let us assume that the two sets have only one common letter $\sigma$, and let $0 \leq l_{1}<\ldots<l_{r} \leq k$ and $k+1 \leq l_{r+1}<\ldots<l_{s} \leq n-1$ be the positions in $w$ such that $w\left(l_{1}\right)=\ldots=w\left(l_{r}\right)=w\left(l_{r+1}\right)=\ldots=w\left(l_{s}\right)=\sigma$.

Since $u_{1} \in \operatorname{preim}_{\Gamma^{(1)} \cup \Sigma^{(2)}}\left(w_{1}\right)$ and $u_{2} \in \operatorname{preim}_{\Gamma^{(2)} \cup \Sigma^{(1)}}\left(w_{2}\right)$ we get that $u\left(l_{1}\right)=\ldots=u\left(l_{r}\right)=x^{(1)}$ and $u\left(l_{r+1}\right)=\ldots=u\left(l_{s}\right)=x^{(2)}$ for some $x^{(1)} \in$ $Z^{(1)} \cup\left\{y^{(1)}\right\}$ and $x^{(2)} \in Z^{(2)} \cup\left\{y^{(2)}\right\}$. If weight $\left(P_{u_{1}}\right) \neq 0 \neq \operatorname{weight}\left(P_{u_{2}}\right)$, by the definition of the list $G_{1}, \ldots, G_{m}$, there is a set $J \subseteq\{1, \ldots, m\}$ such that for every $j \in J$, the path $P_{u^{\prime}}^{\left(G_{j}\right)}$ which is determined by 


$$
\begin{array}{r}
\left(q_{i n_{V_{1}}}^{(1)}, u^{\prime}(0), q_{1}^{(1)}\right) \ldots\left(q_{l_{1}}^{(1)},\left(x^{(1)}, x^{(2)}\right), q_{l_{1}+1}^{(1)}\right) \ldots\left(q_{l_{r}}^{(1)},\left(x^{(1)}, x^{(2)}\right), q_{l_{r}+1}^{(1)}\right) \\
\ldots\left(q_{k-1}^{(1)}, u^{\prime}(k), q_{i n_{V_{2}}}^{(2)}\right)\left(q_{i n_{V_{2}}}^{(2)}, u^{\prime}(k+1), q_{k+1}^{(2)}\right) \ldots\left(q_{l_{r+1}}^{(2)},\left(x^{(1)}, x^{(2)}\right), q_{l_{r+1}+1}^{(2)}\right) \\
\ldots\left(q_{l_{s}}^{(2)},\left(x^{(1)}, x^{(2)}\right), q_{l_{s}+1}^{(2)}\right) \ldots\left(q_{n-1}^{(2)}, u^{\prime}(n-1), q_{\text {ter }_{V_{2}}}^{(2)}\right)
\end{array}
$$

is a path of $A_{G_{j}}$ over $u^{\prime}$, and weight $\left(P_{u^{\prime}}^{\left(G_{j}\right)}\right)=\operatorname{weight}\left(P_{u_{1}}\right)$ weight $\left(P_{u_{2}}\right)$. The word $u^{\prime}$ is obtained by $u$ by replacing the letters $u\left(l_{1}\right), \ldots, u\left(l_{s}\right)$ by $\left(x^{(1)}, x^{(2)}\right)$ and from the remaining letters we replace every occurrence of $y^{(1)}$ and $y^{(2)}$ with $y$. With the same argument, as in the previous case, we obtain $\sum_{1 \leq j \leq m}$ weight $\left(P_{u^{\prime}}^{\left(G_{j}\right)}\right)=$ weight $\left(P_{u_{1}}\right)$ weight $\left(P_{u_{2}}\right)$, and in the obvious way, we define an $h^{(j)} \in V R\left(\Gamma_{G_{j}}\right)$ such that $w \in h^{(j)}\left(u^{\prime}\right)$ for every $j \in J$.

In case the sets $\{w(0), \ldots, w(k)\} \cap\left(\Sigma \backslash \Sigma^{(1)} \cup \Sigma^{(2)}\right)$ and $\{w(k+1), \ldots, w(n-$ $1)\} \cap\left(\Sigma \backslash \Sigma^{(1)} \cup \Sigma^{(2)}\right)$ have more than one common elements, then we similarly merge the labels of the corresponding transitions and construct the paths $P_{u^{\prime}}^{\left(G_{j}\right)}$.

Conversely, let $w \in \Sigma^{+}, u^{\prime(j)} \in \operatorname{preim}_{\Gamma_{G_{j}}}(w)$ for some $1 \leq j \leq m$, and

$$
\begin{array}{r}
P_{u^{\prime}(j)}^{\left(G_{j}\right)}:\left(q_{i n_{V_{1}}}^{(1)}, u^{\prime(j)}(0), q_{1}^{(1)}\right) \ldots\left(q_{k-1}^{(1)}, u^{\prime(j)}(k), q_{i n_{V_{2}}}^{(2)}\right)\left(q_{i n_{V_{2}}}^{(2)}, u^{\prime(j)}(k+1), q_{k+1}^{(2)}\right) \\
\ldots\left(q_{n-1}^{(2)}, u^{\prime(j)}(n-1), q_{t e r_{V_{2}}}^{(2)}\right)
\end{array}
$$

be a path of $A_{G_{j}}$ over $u^{\prime(j)}$. Then, there are two paths $P_{u_{1}}, P_{u_{2}}$ of $A_{\left(\Sigma^{(2)}, V_{1}\right)}^{(1)}$ and $A_{\left(\Sigma(1), V_{2}\right)}^{(2)}$ over $u_{1}$ and $u_{2}$ respectively, where the word $u=u_{1} u_{2}$ is obtained by the word $u^{\prime(j)}$ as follows. For every $0 \leq t \leq k$ we replace every occurrence of $\left(x^{(1)}, x^{(2)}\right)$ (resp. $y$ ) by $x^{(1)}$ (resp. $\left.y^{(1)}\right)$ and for every $k+1 \leq t \leq n-1$ we replace every occurrence of $\left(x^{(1)}, x^{(2)}\right)$ (resp. $y$ ) by $x^{(2)}$ (resp. $\left.y^{(2)}\right)$. Moreover, it is not difficult to show that $u_{1} \in \operatorname{preim}_{\Gamma^{(1)} \cup \Sigma^{(2)}}\left(w_{1}\right), u_{2} \in \operatorname{preim}_{\Gamma^{(2)} \cup \Sigma^{(1)}}\left(w_{2}\right)$, where $w=w_{1} w_{2}$ and weight $\left(P_{u_{1}}\right)$ weight $\left(P_{u_{2}}\right)=$ weight $\left(P_{u^{\prime(j)}}^{\left(G_{j}\right)}\right)$.

We conclude that for every $w \in \Sigma^{+}$it holds

$$
\begin{aligned}
& \left(\left\|\mathcal{A}_{\left(\Sigma^{(2)}, V_{1}\right)}^{(1)}\right\| \cdot\left\|\mathcal{A}_{\left(\Sigma^{(1)}, V_{2}\right)}^{(2)}\right\|, w\right) \\
& =\sum\left\{\left(\left\|\mathcal{A}_{\left(\Sigma^{(2)}, V_{1}\right)}^{(1)}\right\|, w_{1}\right)\left(\left\|\mathcal{A}_{\left(\Sigma^{(1)}, V_{2}\right)}^{(2)}\right\|, w_{2}\right) \mid w=w_{1} w_{2}\right\} \\
& =\sum\left\{\sum_{u_{1} \in \operatorname{preim}_{\Gamma^{(1)} \cup \Sigma^{(2)}\left(w_{1}\right)}\left(\left\|A_{\left(\Sigma^{(2)}, V_{1}\right)}^{(1)}\right\|, u_{1}\right)} \sum_{u_{\Gamma^{(2)} \cup \Sigma^{(1)}\left(w_{2}\right)}}\left(\left\|A_{\left(\Sigma^{(1)}, V_{2}\right)}^{(2)}\right\|, u_{2}\right) \mid w=w_{1} w_{2}\right\}
\end{aligned}
$$




$$
\begin{aligned}
& =\sum\left\{\begin{array}{l}
\left.\sum_{u_{1} \in \operatorname{preim}_{\Gamma^{(1)} \cup \Sigma^{(2)}}\left(w_{1}\right)} \sum_{P_{u_{1}}} \sum_{u_{2} \in \text { preim }_{\Gamma^{(2)} \cup \Sigma^{(1)}}\left(w_{2}\right)} \sum_{P_{u_{2}}} \text { weight }\left(P_{u_{2}}\right) \mid w=w_{1} w_{2}\right\}
\end{array}\right\}
\end{aligned}
$$

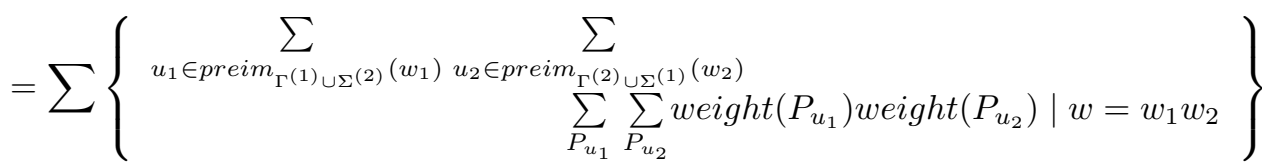

$$
\begin{aligned}
& =\sum_{1 \leq j \leq m} \sum_{u^{\prime(j)} \in \operatorname{preim}_{\Gamma_{G_{j}}}(w)} \sum_{\substack{P_{u^{\prime}(j)}^{\left(G_{j}\right)} \\
\text { weight }}}\left(P_{u^{\prime(j)}}^{\left(G_{j}\right)}\right) \\
& =\sum_{1 \leq j \leq m} \sum_{u^{\prime(j)} \in \operatorname{preim}_{\Gamma_{G_{j}}}(w)}\left(\left\|A_{G_{j}}\right\|, u^{\prime(j)}\right) \\
& =\left(\sum_{1 \leq j \leq m}\left\|\mathcal{A}_{G_{j}}\right\|, w\right)
\end{aligned}
$$

where the fifth equality holds since $K$ is idempotent. Hence, we get

$$
\left\|\mathcal{A}_{\left(\Sigma^{(2)}, V_{1}\right)}^{(1)}\right\| \cdot\left\|\mathcal{A}_{\left(\Sigma^{(1)}, V_{2}\right)}^{(2)}\right\|=\sum_{1 \leq j \leq m}\left\|\mathcal{A}_{G_{j}}\right\|
$$

as required, and our proof is completed.

Proposition 8. The class $\operatorname{VRec}(K, \Sigma)$ is closed under the star operation applied to proper series.

Proof. Let $r \in V \operatorname{Rec}(K, \Sigma)$ be proper and $\mathcal{A}=\langle\Sigma, A\rangle$ a normalized wva, with $A=\left(Q, q_{\text {in }}, w t, q_{\text {ter }}\right)$ over $\Gamma_{A}=\Sigma_{A} \cup Z \cup\{y\}$, accepting $r$. We consider the wva $\mathcal{A}^{\prime}=\left\langle\Sigma, A^{\prime}\right\rangle$ over $\Sigma$ and $K$ with $Q^{\prime}=Q \backslash\left\{q_{\text {ter }}\right\}$ and $A^{\prime}=\left(Q^{\prime}, q_{\text {in }}, w t^{\prime}, q_{\text {in }}\right)$ over $\Gamma_{A}$. The weight assignment mapping $w t^{\prime}: Q^{\prime} \times \Gamma_{A} \times Q^{\prime} \rightarrow K$ is defined for every $q, q^{\prime} \in Q^{\prime}, \sigma \in \Gamma_{A}$ as follows:

$$
w t^{\prime}\left(\left(q, \sigma, q^{\prime}\right)\right)=\left\{\begin{array}{ll}
w t\left(\left(q, \sigma, q^{\prime}\right)\right) & \text { if } q^{\prime} \neq q_{i n} \\
w t\left(\left(q, \sigma, q_{t e r}\right)\right) & \text { if } q^{\prime}=q_{\text {in }}
\end{array} .\right.
$$

By standard arguments on weighted automata, we can show that $\left\|A^{\prime}\right\|=\|A\|^{*}$. Moreover, for every $w \in \Sigma^{*}$ we have

$$
\begin{aligned}
\left(\left\|\mathcal{A}^{\prime}\right\|, w\right) & =\sum_{u \in \text { preim }_{\Gamma_{A}}(w)}\left(\left\|A^{\prime}\right\|, u\right) \\
& =\sum_{u \in \text { preim }_{\Gamma_{A}}(w)}\left(\|A\|^{*}, u\right) \\
& =\sum_{u \in \text { preim }_{\Gamma_{A}}(w)} \sum_{n \geq 0, u=u_{1} \ldots u_{n}}\left(\left(\|A\|, u_{1}\right) \cdot \ldots \cdot\left(\|A\|, u_{n}\right)\right)
\end{aligned}
$$




$$
\begin{aligned}
& =\sum_{n \geq 0, w=w_{1} \ldots w_{n}} \sum_{u_{i} \in \text { preim }_{\Gamma_{A}}\left(w_{i}\right)}\left(\left(\|A\|, u_{1}\right) \cdot \ldots \cdot\left(\|A\|, u_{n}\right)\right) \\
& =\sum_{n \geq 0, w=w_{1} \ldots w_{n}} \sum_{u_{1} \in \text { preim }_{\Gamma_{A}}\left(w_{1}\right)}\left(\|A\|, u_{1}\right) \cdot \ldots \cdot \sum_{u_{n} \in \text { preim }_{\Gamma_{A}}\left(w_{n}\right)}\left(\|A\|, u_{n}\right) \\
& =\sum_{n \geq 0, w=w_{1} \ldots w_{n}}\left(r, w_{1}\right) \cdot \ldots \cdot\left(r, w_{n}\right) \\
& =\left(r^{*}, w\right)
\end{aligned}
$$

which implies that $\left\|\mathcal{A}^{\prime}\right\|=r^{*}$ hence, $r^{*} \in V \operatorname{Rec}(K, \Sigma)$ and our proof is completed.

Proposition 9. The class $V \operatorname{Rec}(K, \Sigma)$ is closed under the shuffle product.

Proof. Let $r^{(i)} \in V \operatorname{Rec}(K, \Sigma)$ with $i=1,2$. We consider the proper series $r^{(1)}, r^{(2)}$ over $\Sigma$ and $K$ defined, for every $w \in \Sigma^{*}$, by

$$
\begin{aligned}
& \text { - }\left(r^{\prime(1)}, w\right)=\left\{\begin{array}{ll}
\left(r^{(1)}, w\right) & \text { if } w \in \Sigma^{+} \\
0 & \text { otherwise }
\end{array},\right. \text { and } \\
& \text { - }\left(r^{\prime(2)}, w\right)= \begin{cases}\left(r^{(2)}, w\right) & \text { if } w \in \Sigma^{+} \\
0 & \text { otherwise. }\end{cases}
\end{aligned}
$$

Then $r^{(1)} ш r^{(2)}=r^{(1)} ш r^{(2)}+\left(r^{(1)}, \varepsilon\right) r^{(2)}+r^{(1)}\left(r^{(2)}, \varepsilon\right)+\left(r^{(1)}, \varepsilon\right)\left(r^{(2)}, \varepsilon\right) \bar{\varepsilon}$. By Propositions 2, 4, and 5 it suffices to show that $r^{\prime(1)} \uplus r^{(2)} \in \operatorname{VRec}(K, \Sigma)$. By Proposition 3, we can construct normalized wva $\mathcal{A}^{(i)}=\left\langle\Sigma, A^{(i)}\right\rangle$ with $A^{(i)}=$ $\left(Q^{(i)}, q_{\text {in }}^{(i)}, w t^{(i)}, q_{\text {ter }}^{(i)}\right)$ over $\Gamma^{(i)}=\Sigma^{(i)} \cup Z^{(i)} \cup\left\{y^{(i)}\right\}$ and $K$, accepting respectively $r^{(i)}$, with $i=1,2$. Without any loss, we assume that $Q^{(1)} \cap Q^{(2)}=\emptyset$ and $\left(Z^{(1)} \cup\left\{y^{(1)}\right\}\right) \cap\left(Z^{(2)} \cup\left\{y^{(2)}\right\}\right)=\emptyset$. We consider the wva $\mathcal{A}_{\left(\Sigma^{(2)}, V_{1}\right)}^{(1)}=$ $\left\langle\Sigma, A_{\left(\Sigma^{(2)}, V_{1}\right)}^{(1)}\right\rangle$ and $\mathcal{A}_{\left(\Sigma^{(1)}, V_{2}\right)}^{(2)}=\left\langle\Sigma, A_{\left(\Sigma^{(1)}, V_{2}\right)}^{(2)}\right\rangle$ determined by the procedure before Lemma 1. By Proposition 3 and Lemma 1 these wva can be also assumed to be normalized hence, let $A_{\left(\Sigma^{(2)}, V_{1}\right)}^{(1)}=\left(Q_{V_{1}}^{(1)}, q_{i n_{V_{1}}}^{(1)}, w t_{V_{1}}^{(1)}, q_{\text {ter }_{V_{1}}}^{(1)}\right)$ over $\Gamma^{(1)} \cup \Sigma^{(2)}$ and $A_{\left(\Sigma^{(1)}, V_{2}\right)}^{(2)}=\left(Q_{V_{2}}^{(2)}, q_{i n_{V_{2}}}^{(2)}, w t_{V_{2}}^{(2)}, q_{t e r_{V_{2}}}^{(2)}\right)$ over $\Gamma^{(2)} \cup \Sigma^{(1)}$. Moreover, without any loss, we assume that $Q_{V_{1}}^{(1)} \cap Q_{V_{2}}^{(2)}=\emptyset$. We let $y=\left(y^{(1)}, y^{(2)}\right)$ and consider the set $H=\left(Z^{(1)} \cup\left\{y^{(1)}\right\}\right) \times\left(Z^{(2)} \cup\left\{y^{(2)}\right\}\right) \backslash\{y\}$ and a maximal subset $G \subseteq H \cup Z^{(1)} \cup Z^{(2)}$ satisfying the following condition: every element of $Z^{(1)}$ (resp. of $Z^{(2)}$ ) occurs either in at most one pair of $H$ as a left (resp. as a right) coordinate, or as a single element of $G$. Assume that $G_{1}, \ldots, G_{m}$ is an enumeration of all such sets. We let $Q=Q_{V_{1}}^{(1)} \times Q_{V_{2}}^{(2)}, \Gamma_{G_{j}}=\Sigma^{(1)} \cup \Sigma^{(2)} \cup G_{j} \cup\{y\}$, for every $1 \leq j \leq m$, and consider the normalized wva $\mathcal{A}_{G_{j}}=\left\langle\Sigma, A_{G_{j}}\right\rangle$ over $\Sigma$ and $K$ 
with $A_{G_{j}}=\left(Q,\left(q_{i n_{V_{1}}}^{(1)}, q_{i n_{V_{2}}}^{(2)}\right), w t_{G_{j}},\left(q_{\text {ter }_{V_{1}}}^{(1)}, q_{\text {ter }_{V_{2}}}^{(2)}\right)\right)$ over $\Gamma_{G_{j}}$, where the weight assignment mapping $w t_{G_{j}}$ is defined for every $1 \leq j \leq m$ as follows:

$$
\begin{aligned}
& w t_{G_{j}}\left(\left(\left(q^{(1)}, q^{(2)}\right), \sigma,\left(q^{\prime(1)}, q^{\prime(2)}\right)\right)\right)= \\
& \begin{cases}w t_{V_{1}}^{(1)}\left(\left(q^{(1)}, \sigma, q^{\prime(1)}\right)\right) & \text { if } q^{(2)}=q^{\prime(2)} \text { and } \sigma \in \Sigma^{(1)} \cup \Sigma^{(2)} \cup\left(Z^{(1)} \cap G_{j}\right) \\
w t_{V_{2}}^{(2)}\left(\left(q^{(2)}, \sigma, q^{\prime(2)}\right)\right) & \text { if } q^{(1)}=q^{\prime(1)} \text { and } \sigma \in \Sigma^{(1)} \cup \Sigma^{(2)} \cup\left(Z^{(2)} \cap G_{j}\right) \\
w t_{V_{1}}^{(1)}\left(\left(q^{(1)}, x^{(1)}, q^{\prime(1)}\right)\right) & \text { if } q^{(2)}=q^{\prime(2)} \text { and } \sigma=\left(x^{(1)}, x^{(2)}\right) \in G_{j} \cup\{y\} \\
w t_{V_{2}}^{(2)}\left(\left(q^{(2)}, x^{(2)}, q^{(2)}\right)\right) & \text { if } q^{(1)}=q^{\prime(1)} \text { and } \sigma=\left(x^{(1)}, x^{(2)}\right) \in G_{j} \cup\{y\} \\
0 & \text { otherwise }\end{cases}
\end{aligned}
$$

for every $\left(q^{(1)}, q^{(2)}\right),\left(q^{\prime(1)}, q^{\prime(2)}\right) \in Q, \sigma \in \Gamma_{G_{j}}$.

Next, we show that $\left\|\mathcal{A}_{\left(\Sigma^{(2)}, V_{1}\right)}^{(1)}\right\| ш\left\|\mathcal{A}_{\left(\Sigma^{(1)}, V_{2}\right)}^{(2)}\right\|=\sum_{1 \leq j \leq m}\left\|\mathcal{A}_{G_{j}}\right\|$.

For this let $w, w_{1}=w_{1}(0) \ldots w_{1}\left(n_{1}-1\right), w_{2}=w_{2}(0) \ldots w_{2}\left(n_{2}-1\right) \in \Sigma^{+}$such that $w \in w_{1} \amalg w_{2}$, and $u_{1} \in \operatorname{preim}_{\Gamma^{(1)} \cup \Sigma^{(2)}}\left(w_{1}\right), u_{2} \in \operatorname{preim}_{\Gamma^{(2)} \cup \Sigma^{(1)}}\left(w_{2}\right)$. Hence, there exist valid relabelings $h^{(1)} \in V R\left(\Gamma^{(1)} \cup \Sigma^{(2)}\right)$ and $h^{(2)} \in V R\left(\Gamma^{(2)} \cup \Sigma^{(1)}\right)$ such that $w_{1} \in h^{(1)}\left(u_{1}\right), w_{2} \in h^{(2)}\left(u_{2}\right)$. We consider a path

$$
P_{u_{1}}:\left(q_{i n_{V_{1}}}^{(1)}, u_{1}(0), q_{1}^{(1)}\right) \ldots\left(q_{n_{1}-1}^{(1)}, u_{1}\left(n_{1}-1\right), q_{\text {ter }_{V_{1}}}^{(1)}\right)
$$

of $A_{\left(\Sigma^{(2)}, V_{1}\right)}^{(1)}$ over $u_{1}$ and a path

$$
P_{u_{2}}:\left(q_{i n_{V_{2}}}^{(2)}, u_{2}(0), q_{1}^{(2)}\right) \ldots\left(q_{n_{2}-1}^{(2)}, u_{2}\left(n_{2}-1\right), q_{\text {ter }_{V_{2}}}^{(2)}\right)
$$

of $A_{\left(\Sigma^{(1)}, V_{2}\right)}^{(2)}$ over $u_{2}$. We distinguish the following cases.

- The sets $\left\{w_{1}(0), \ldots, w_{1}\left(n_{1}-1\right)\right\} \cap\left(\Sigma \backslash\left(\Sigma^{(1)} \cup \Sigma^{(2)}\right)\right)$ and $\left\{w_{2}(0), \ldots, w_{2}\left(n_{2}-\right.\right.$ $1)\} \cap\left(\Sigma \backslash\left(\Sigma^{(1)} \cup \Sigma^{(2)}\right)\right)$ are disjoint. Then, if weight $\left(P_{u_{1}}\right) \neq 0 \neq$ weight $\left(P_{u_{2}}\right)$, by the definition of the list $G_{1}, \ldots, G_{m}$, there is a set $J \subseteq\{1, \ldots, m\}$ such that for every $j \in J$ there is a path $P_{u}^{\left(G_{j}\right)}$ of $A_{G_{j}}$ over $u$, for $u \in\left(u_{1} \amalg u_{2}\right) \cap$ $\operatorname{preim}_{\Gamma_{G_{j}}}(w)$ with weight $\left(P_{u}^{\left(G_{j}\right)}\right)=\operatorname{weight}\left(P_{u_{1}}\right)$ weight $\left(P_{u_{2}}\right)$. Since $K$ is idempotent it holds $\sum_{j \in J}$ weight $\left(P_{u}^{\left(G_{j}\right)}\right)=$ weight $\left(P_{u_{1}}\right)$ weight $\left(P_{u_{2}}\right)$ and thus $\sum_{1 \leq j \leq m}$ weight $\left(P_{u}^{\left(G_{j}\right)}\right)=$ weight $\left(P_{u_{1}}\right)$ weight $\left(P_{u_{2}}\right)$.

- The sets $\left\{w_{1}(0), \ldots, w_{1}\left(n_{1}-1\right)\right\} \cap\left(\Sigma \backslash\left(\Sigma^{(1)} \cup \Sigma^{(2)}\right)\right)$ and $\left\{w_{2}(0), \ldots, w_{2}\left(n_{2}-\right.\right.$ $1)\} \cap\left(\Sigma \backslash\left(\Sigma^{(1)} \cup \Sigma^{(2)}\right)\right)$ are not disjoint. Moreover, for simplicity, we assume that the two sets have only one common letter $\sigma$, and let $0 \leq l_{1}<\ldots<$ $l_{r} \leq n_{1}-1$ and $0 \leq g_{1}<\ldots<g_{s} \leq n_{2}-1$ be the positions in $w_{1}, w_{2}$ respectively, such that $w_{1}\left(l_{1}\right)=\ldots=w_{1}\left(l_{r}\right)=w_{2}\left(g_{1}\right)=\ldots=w_{2}\left(g_{s}\right)=\sigma$. 
Since $u_{1} \in \operatorname{preim}_{\Gamma^{(1)} \cup \Sigma^{(2)}}\left(w_{1}\right)$ and $u_{2} \in \operatorname{preim}_{\Gamma^{(2)} \cup \Sigma^{(1)}}\left(w_{2}\right)$ we get that $u_{1}\left(l_{1}\right)=\ldots=u_{1}\left(l_{r}\right)=x^{(1)}$ and $u_{2}\left(g_{1}\right)=\ldots=u_{2}\left(g_{s}\right)=x^{(2)}$ for some $x^{(1)} \in$ $Z^{(1)} \cup\left\{y^{(1)}\right\}$ and $x^{(2)} \in Z^{(2)} \cup\left\{y^{(2)}\right\}$. If weight $\left(P_{u_{1}}\right) \neq 0 \neq \operatorname{weight}\left(P_{u_{2}}\right)$, by the definition of the list $G_{1}, \ldots, G_{m}$, there is a set $J \subseteq\{1, \ldots, m\}$ such that for every $j \in J$ there is a path $P_{u^{\prime}}^{\left(G_{j}\right)}$ of $A_{G_{j}}$ over $u^{\prime}$, where $u^{\prime}$ is obtained by $u$ by replacing $x^{(1)}$ (resp. $\left.x^{(2)}\right)$ in $u_{1}$ (resp. $u_{2}$ ) at the positions $l_{1}, \ldots, l_{r}$ (resp. $\left.g_{1}, \ldots, g_{s}\right)$ by the pair $\left(x^{(1)}, x^{(2)}\right)$, and from the remaining letters we replace every occurrence of $y^{(1)}$ and $y^{(2)}$ with $y$, for $u \in u_{1} \amalg u_{2}$. Again, we have weight $\left(P_{u^{\prime}}^{\left(G_{j}\right)}\right)=\operatorname{weight}\left(P_{u_{1}}\right)$ weight $\left(P_{u_{2}}\right)$ and hence, $\sum_{1 \leq j \leq m}$ weight $\left(P_{u^{\prime}}^{\left(G_{j}\right)}\right)=$ weight $\left(P_{u_{1}}\right)$ weight $\left(P_{u_{2}}\right)$. On the other hand, it is trivially shown that $u^{\prime} \in \operatorname{preim}_{\Gamma_{G_{j}}}(w)$.

Conversely, keeping the previous notations, for every $w \in \Sigma^{+}, u^{\prime} \in \operatorname{preim}_{\Gamma_{G_{j}}}(w)$ for some $1 \leq j \leq m$, there are $u_{1} \in \operatorname{preim}_{\Gamma^{(1)} \cup \Sigma^{(2)}}\left(w_{1}\right), u_{2} \in \operatorname{preim}_{\Gamma^{(2)} \cup \Sigma^{(1)}}\left(w_{2}\right)$ with $w \in w_{1} ш w_{2}$, such that for every path $P_{u^{\prime}}^{\left(G_{j}\right)}$ of $A_{G_{j}}$ over $u^{\prime}$, there are paths $P_{u_{1}}$ of $A_{\left(\Sigma^{(2)}, V_{1}\right)}^{(1)}$ over $u_{1}$ and $P_{u_{2}}$ of $A_{\left(\Sigma^{(1)}, V_{2}\right)}^{(2)}$ over $u_{2}$, with weight $\left(P_{u^{\prime}}^{\left(G_{j}\right)}\right)=$ weight $\left(P_{u_{1}}\right)$ weight $\left(P_{u_{2}}\right)$. Using the same as above argument, we can show that $\sum_{1 \leq j \leq m}$ weight $\left(P_{u^{\prime}}^{\left(G_{j}\right)}\right)=\operatorname{weight}\left(P_{u_{1}}\right)$ weight $\left(P_{u_{2}}\right)$.

Now for every $w_{1}, w_{2} \in \Sigma^{+}$, it holds

$$
\begin{aligned}
& \left(\left\|\mathcal{A}_{\left(\Sigma^{(2)}, V_{1}\right)}^{(1)}\right\|, w_{1}\right)\left(\left\|\mathcal{A}_{\left(\Sigma^{(1)}, V_{2}\right)}^{(2)}\right\|, w_{2}\right) \\
& =\sum_{u_{1} \in \text { preim }_{\Gamma^{(1)} \cup \Sigma^{(2)}\left(w_{1}\right)}}\left(\left\|A_{\left(\Sigma^{(2)}, V_{1}\right)}^{(1)}\right\|, u_{1}\right) \sum_{u_{2} \in \text { preim }_{\Gamma^{(2)} \cup \Sigma^{(1)}}\left(w_{2}\right)}\left(\left\|A_{\left(\Sigma^{(1)}, V_{2}\right)}^{(2)}\right\|, u_{2}\right) \\
& =\sum_{u_{1} \in \text { preim }_{\Gamma^{(1)} \cup \Sigma^{(2)}}} \sum_{\left.w_{1}\right)} \text { weight }\left(P_{u_{1}}\right) \sum_{u_{2} \in \operatorname{preim}_{\Gamma^{(2)} \cup \Sigma^{(1)}}\left(w_{2}\right)} \sum_{P_{u_{2}}} \operatorname{weight}\left(P_{u_{2}}\right) \\
& =\sum_{u_{1} \in \operatorname{preim}_{\Gamma^{(1)} \cup \Sigma^{(2)}}} \sum_{\left.w_{1}\right)} \sum_{u_{2} \in \operatorname{preim}_{\Gamma^{(2)} \cup \Sigma^{(1)}}} \sum_{\left.w_{2}\right)} \sum_{P_{u_{1}}} \text { weight }\left(P_{u_{1}}\right) \text { weight }\left(P_{u_{2}}\right) \text {. }
\end{aligned}
$$

Hence, for every $w \in \Sigma^{+}$, we get

$$
\begin{aligned}
& \left(\left\|\mathcal{A}_{\left(\Sigma^{(2)}, V_{1}\right)}^{(1)}\right\| w\left\|\mathcal{A}_{\left(\Sigma^{(1)}, V_{2}\right)}^{(2)}\right\|, w\right) \\
& \left.=\sum_{\substack{w_{1}, w_{2} \in \Sigma^{+} \\
w \in w_{1} \amalg w_{2}}}\left(\left\|\mathcal{A}_{\left(\Sigma^{(2)}, V_{1}\right)}^{(1)}\right\|, w_{1}\right)\left(\left\|\mathcal{A}_{\left(\Sigma^{(1)}, V_{2}\right)}^{(2)}\right\|, w_{2}\right)\right) \\
& =\sum_{\substack{w_{1}, w_{2} \in \Sigma^{+} \\
w \in w_{1} \amalg w_{2}}} \sum_{u_{1} \in \operatorname{preim}_{\Gamma^{(1)} \cup \Sigma^{(2)}}\left(w_{1}\right)}\left(\left\|A_{\left(\Sigma^{(2)}, V_{1}\right)}^{(1)}\right\|, u_{1}\right)
\end{aligned}
$$




$$
\begin{aligned}
& \sum_{u_{2} \in \operatorname{preim}_{\Gamma^{(2)} \cup \Sigma^{(1)}}\left(w_{2}\right)}\left(\left\|A_{\left(\Sigma^{(1)}, V_{2}\right)}^{(2)}\right\|, u_{2}\right) \\
& =\sum_{\substack{w_{1}, w_{2} \in \Sigma^{+} \\
w \in w_{1} \amalg w_{2}}} \sum_{u_{1} \in \text { preim }_{\Gamma^{(1)} \cup \Sigma^{(2)}}} \sum_{\left.w_{1}\right)} \text { weight }\left(P_{u_{1}}\right) \\
& \sum_{u_{2} \in \operatorname{preim}_{\Gamma^{(2)} \cup \Sigma^{(1)}}} \sum_{\left.w_{2}\right)} \operatorname{weight}\left(P_{u_{2}}\right) \\
& =\sum_{\substack{w_{1}, w_{2} \in \Sigma^{+} \\
w \in w_{1} \amalg w_{2}}} \sum_{u_{1} \in \text { preim }_{\Gamma^{(1)} \cup \Sigma^{(2)}}\left(w_{1}\right)} \sum_{u_{2} \in \text { preim }_{\Gamma^{(2)} \cup \Sigma^{(1)}}\left(w_{2}\right)} \\
& \sum_{P_{u_{1}}} \sum_{P_{u_{2}}} \text { weight }\left(P_{u_{1}}\right) \text { weight }\left(P_{u_{2}}\right) \\
& =\sum_{1 \leq j \leq m} \sum_{u \in \text { preim }_{\Gamma_{G_{j}}}(w)} \sum_{P_{u}^{\left(G_{j}\right)}} \text { weight }\left(P_{u}^{\left(G_{j}\right)}\right) \\
& =\left(\sum_{1 \leq j \leq m}\left\|\mathcal{A}_{G_{j}}\right\|, w\right)
\end{aligned}
$$

which implies that $\left\|\mathcal{A}_{\left(\Sigma^{(2)}, V_{1}\right)}^{(1)}\right\| w\left\|\mathcal{A}_{\left(\Sigma^{(1)}, V_{2}\right)}^{(2)}\right\|=\sum_{1 \leq j \leq m}\left\|\mathcal{A}_{G_{j}}\right\|$ hence, $r^{\prime(1)} ш$ $r^{\prime(2)}=\sum_{1 \leq j \leq m}\left\|\mathcal{A}_{G_{j}}\right\|$. Therefore, by Proposition 4, we conclude that $r^{(1)} w r^{(2)} \in$ $V \operatorname{Rec}(K, \Sigma)$, as required.

Remark 1. The definition of the weight assignment mappings $w t_{G_{j}}$ for $1 \leq j \leq m$, in the above proof, is not completely right. More precisely, it may happen that there are $q^{(1)} \in Q_{V_{1}}^{(1)}, q^{(2)} \in Q_{V_{2}}^{(2)}$ and $\sigma \in\left(\Sigma^{(1)} \cap \Sigma^{(2)}\right)\left(\right.$ resp. $\left.\sigma=\left(x^{(1)}, x^{(2)}\right) \in G_{j} \cup\{y\}\right)$ such that $w t_{V_{1}}^{(1)}\left(\left(q^{(1)}, \sigma, q^{(1)}\right)\right)=k_{1} \neq 0, w t_{V_{2}}^{(2)}\left(\left(q^{(2)}, \sigma, q^{(2)}\right)\right)=k_{2} \neq 0$ with $k_{1} \neq k_{2}\left(\operatorname{resp} . w t_{V_{1}}^{(1)}\left(\left(q^{(1)}, x^{(1)}, q^{(1)}\right)\right)=k_{1} \neq 0, w t_{V_{2}}^{(2)}\left(\left(q^{(2)}, x^{(2)}, q^{(2)}\right)\right)=k_{2} \neq 0\right.$ with $\left.k_{1} \neq k_{2}\right)$. Then, the value $w_{G_{j}}\left(\left(\left(q^{(1)}, q^{(2)}\right), \sigma,\left(q^{(1)}, q^{(2)}\right)\right)\right)$ is not welldefined $^{3}$. Therefore, if this is the case, for every such pair of states $\left(q^{(1)}, q^{(2)}\right)$ and $\sigma \in\left(\Sigma^{(1)} \cap \Sigma^{(2)}\right) \cup G_{j} \cup\{y\}$, we introduce a new state $r$ and we set

$$
\begin{aligned}
& -w t_{G_{j}}\left(\left(\left(q^{(1)}, q^{(2)}\right), \sigma,\left(q^{(1)}, q^{(2)}\right)\right)\right)=k_{1}, \\
& \text { - } w t_{G_{j}}\left(\left(\left(q^{(1)}, q^{(2)}\right), \sigma, r\right)\right)=k_{1}, \\
& \text { - } w t_{G_{j}}\left(\left(r, \sigma,\left(q^{(1)}, q^{(2)}\right)\right)\right)=k_{2},
\end{aligned}
$$

\footnotetext{
${ }^{3}$ Other authors proving the closure of the class of recognizable series under the shuffle product have also ignored this case (cf. for instance [34]).
} 


$$
\begin{aligned}
& \text { - } w t_{G_{j}}((r, \sigma, r))=k_{2} \text {, and } \\
& \text { - } w t_{G_{j}}\left(\left(\left(q^{\prime(1)}, q^{\prime(2)}\right), \sigma^{\prime}, r\right)\right)=w t_{G_{j}}\left(\left(\left(q^{\prime(1)}, q^{\prime(2)}\right), \sigma^{\prime},\left(q^{(1)}, q^{(2)}\right)\right)\right), \\
& \text { - } w t_{G_{j}}\left(\left(r, \sigma^{\prime},\left(q^{\prime(1)}, q^{\prime(2)}\right)\right)\right)=w t_{G_{j}}\left(\left(\left(q^{(1)}, q^{(2)}\right), \sigma^{\prime},\left(q^{\prime(1)}, q^{\prime(2)}\right)\right)\right),
\end{aligned}
$$

for every $q^{\prime(1)} \in Q_{V_{1}}^{(1)}, q^{\prime(2)} \in Q_{V_{2}}^{(2)}, \sigma^{\prime} \in \Gamma_{G_{j}}$.

The following theorem summarizes the results of this section.

Theorem 1. Let $K$ be a commutative and idempotent semiring and $\Sigma$ an infinite alphabet. Then the class of v-recognizable series over $\Sigma$ and $K$ is closed under sum, and under scalar, Hadamard, Cauchy and shuffle products, and under star operation applied to proper series.

\section{$5 \quad$ Rational series over infinite alphabets}

In this section, we extend the notion of rational series over the infinite alphabet $\Sigma$ and the semiring $K^{4}$. In fact, we state a Kleene-Schützenberger type result for v-recognizable series over $\Sigma$ and $K$. For this, we define the notion of rationality for series over $\Sigma$ in the same way we did it for v-recognizable series. A similar approach for defining regular expressions over infinite alphabets has been followed in $[1,25]$. Firstly, we recall the concept of rational series over finite alphabets. Let $\Delta$ be a finite alphabet. The $\operatorname{class} \operatorname{Rat}(K, \Delta)$ of rational series over $\Delta$ and $K$ is the least class of series containing the polynomials over $\Delta$ and $K$ and being closed under sum, Cauchy product, and star operation applied to proper series. The subsequent result is the fundamental theorem of Schützenberger stating the coincidence of rational and recognizable series.

Theorem 2. [35, 18, 34] Let $K$ be a semiring and $\Delta$ a finite alphabet. Then a series $s \in K\left\langle\left\langle\Delta^{*}\right\rangle\right\rangle$ is recognizable iff it is rational.

Definition 3. A series $s$ over $\Sigma$ and $K$ is called $\mathrm{v}$-rational if there is a subalphabet $\Gamma \subseteq_{\text {fin }} \Sigma$ and a rational series $s^{\prime}$ over $\Delta=\Gamma \cup Z \cup\{y\}$ and $K$ such that

$$
(s, w)=\sum_{u \in \text { preim }_{\Delta}(w)}\left(s^{\prime}, u\right)
$$

for every $w \in \Sigma^{*}$.

Now we discuss why we adopted the above definition for rational series over infinite alphabets. One could think of alternative definitions, more precisely, by defining rational series over the infinite alphabet $\Sigma$ in the same way we do it for rational series over finite alphabets. It is not difficult to see that such a consideration should

\footnotetext{
${ }^{4}$ In this section we can relax the commutativity property of $K$.
} 
not derive an expressively equivalent notion to wva. Consider for instance the normalized wva $\mathcal{A}=\langle\Sigma, A\rangle$ where $A=\left(\left\{q_{\text {in }}, q_{\text {ter }}\right\}, q_{\text {in }}, w t, q_{t e r}\right)$ with $\Sigma_{A}=\{a\}$ and $Z=\{z\}$. The only non-zero assignment of $w t$ is given by $w t\left(\left(q_{i n}, z, q_{\text {ter }}\right)\right)=k \neq 0$. Then trivially, $\|\mathcal{A}\|=\sum_{a^{\prime} \in \Sigma \backslash\{a\}} k a^{\prime}$ and it is not difficult to see that this series is not rational in the sense of rational series over finite alphabets. Even if we should consider our rational series to contain, by definition, series of the above form, then still this is not sufficient. For instance let us consider the normalized wva $\mathcal{B}=\langle\Sigma, B\rangle$ where $B=\left(\left\{p_{\text {in }}, p, p_{\text {ter }}\right\}, p_{\text {in }}, w t, p_{\text {ter }}\right)$ with $\Sigma_{B}=\{b\}, Z=\left\{z, z^{\prime}\right\}$ and non-zero weights $w t\left(\left(p_{i n}, z, p\right)\right)=k, w t\left(\left(p, z^{\prime}, p_{\text {ter }}\right)\right)=k^{\prime}$. Then it is easily obtained that

$$
\|\mathcal{B}\|=\sum_{\substack{a, a^{\prime} \in \Sigma \backslash\{b\} \\ a \neq a^{\prime}}} k k^{\prime} a a^{\prime} .
$$

On the other hand, the Cauchy product of the series $\sum_{a \in \Sigma \backslash\{b\}} k a \sum_{a^{\prime} \in \Sigma \backslash\{b\}} k^{\prime} a^{\prime}$ clearly differs from $\|\mathcal{B}\|$.

Next, we state our Kleene-Schützenberger type theorem for series over $\Sigma$ and $K$.

Theorem 3. Let $K$ be a semiring and $\Sigma$ an infinite alphabet. Then a series $s \in K\left\langle\left\langle\Sigma^{*}\right\rangle\right\rangle$ is v-recognizable iff it is v-rational.

Proof. Let $s \in V \operatorname{Rec}(K, \Sigma)$. Then, there exists a wva $\mathcal{A}=\langle\Sigma, A\rangle$ where $A$ is a weighted automaton over $\Gamma_{A}=\Sigma_{A} \cup Z \cup\{y\}$ such that

$$
s=\|\mathcal{A}\|=\sum_{u \in \text { preim }_{\Gamma_{A}}(w)}(\|A\|, u) .
$$

By Theorem 2 the recognizable series $\|A\|$ over $\Gamma_{A}$ and $K$ is also rational. This implies that $s$ is v-rational. By a similar argument, we show that if $s$ is v-rational, then it is also v-recognizable, and this concludes our proof.

\section{Weighted monadic second order logic over infi- nite alphabets}

Droste and Gastin in their seminal paper [12] (cf. also [13]), introduced a weighted monadic second order logic ( $M S O$ logic for short) and proved in the quantitative setup the fundamental result of Büchi [7], Elgot [17], and Trakhtenbrot [41] relating recognizable and $M S O$-definable languages. More precisely, they determined two fragments of their weighted $M S O$ logic, namely the restricted, and the existential restricted one, and proved that the classes of series defined by sentences in these two fragments coincide with the class of recognizable series over a finite alphabet and a commutative semiring. We would like to extend this result for the class of v-recognizable series. For this, we introduce a weighted $M S O$ logic over the infinite alphabet $\Sigma$ and the commutative semiring $K$. 
Firstly we recall, for the reader's convenience, the basic definitions of weighted $M S O$ logic (cf. [12, 13]) by adopting the notations of [19].

Let $\Delta$ be a finite alphabet. The syntax of $M S O$ logic formulas over $\Delta$ is given by the grammar

$$
\phi::=\text { true }\left|P_{a}(x)\right| x \leq x^{\prime}|x \in X| \neg \phi|\phi \vee \phi| \exists x \cdot \phi \mid \exists X . \phi
$$

where $a \in \Delta$ and we let false $=\neg$ true. The set $\operatorname{free}(\phi)$ of free variables of an $M S O$ logic formula $\phi$ is defined as usual. In order to define the semantics of $M S O$ logic formulas we need the notions of the extended alphabet and valid assignment (cf. for instance [40]). Let $\mathcal{V}$ be a finite set of first and second order variables. For every word $u=u(0) \ldots u(n-1) \in \Delta^{*}$ we let $\operatorname{Dom}(u)=\{0, \ldots, n-1\}$. A $(\mathcal{V}, u)$ assignment $\sigma$ is a mapping associating first order variables from $\mathcal{V}$ to elements of $\operatorname{Dom}(u)$, and second order variables from $\mathcal{V}$ to subsets of $\operatorname{Dom}(u)$. If $x$ is a first order variable and $i \in \operatorname{Dom}(u)$, then $\sigma[x \rightarrow i]$ denotes the $(\mathcal{V} \cup\{x\}, u)$-assignment which associates $i$ to $x$ and coincides with $\sigma$ on $\mathcal{V} \backslash\{x\}$. For a second order variable $X$ and $I \subseteq \operatorname{Dom}(u)$, the notation $\sigma[X \rightarrow I]$ has a similar meaning.

We shall encode pairs of the form $(u, \sigma)$, where $u \in \Delta^{*}$ and $\sigma$ is a $(\mathcal{V}, u)$ assignment, using the extended alphabet $\Delta_{\mathcal{V}}=\Delta \times\{0,1\}^{\mathcal{V}}$. Indeed, every word in $\Delta_{\mathcal{V}}^{*}$ can be considered as a pair $(u, \sigma)$ where $u$ is the projection over $\Delta$ and $\sigma$ is the projection over $\{0,1\}^{\mathcal{V}}$. Then $\sigma$ is a valid assignment if for every first order variable $x \in \mathcal{V}$ the $x$-row contains exactly one 1 . In this case, $\sigma$ is the $(\mathcal{V}, u)$-assignment such that for every first order variable $x \in \mathcal{V}, \sigma(x)$ is the position of the 1 on the $x$-row, and for every second order variable $X \in \mathcal{V}, \sigma(X)$ is the set of positions labelled with 1 along the $X$-row. It is not difficult to see that the language

$$
N_{\mathcal{V}}=\left\{(u, \sigma) \in \Delta_{\mathcal{V}}^{*} \mid \sigma \text { is a valid }(\mathcal{V}, u) \text {-assignment }\right\}
$$

is recognizable.

For every $(u, \sigma) \in \mathcal{N}_{\mathcal{V}}$ we define the satisfaction relation $(u, \sigma) \models \phi$ by induction on the structure of $\phi$, as follows:

- $(u, \sigma) \models$ true,

- $(u, \sigma) \models P_{a}(x)$ iff $u(\sigma(x))=a$,

- $(u, \sigma) \models x \leq x^{\prime}$ iff $\sigma(x) \leq \sigma\left(x^{\prime}\right)$,

- $(u, \sigma) \models x \in X$ iff $\sigma(x) \in \sigma(X)$,

- $(u, \sigma) \models \neg \phi$ iff $(u, \sigma) \not \models \phi$,

- $(u, \sigma) \models \phi \vee \phi^{\prime} \quad$ iff $(u, \sigma) \models \phi$ or $(u, \sigma) \models \phi^{\prime}$,

- $(u, \sigma) \models \exists x \cdot \phi$ iff there exists an $i \in \operatorname{Dom}(u)$ such that $(u, \sigma[x \rightarrow i) \models \phi$,

- $(u, \sigma) \models \exists X$. $\phi$ iff there exists an $I \subseteq \operatorname{Dom}(u)$ such that $(u, \sigma[X \rightarrow I) \models \phi$.

If $(u, \sigma) \in \Delta_{\mathcal{V}}^{*} \backslash \mathcal{N}_{\mathcal{V}}$, then we let $(u, \sigma) \not=\phi$. 
Definition 4. The syntax of formulas of the weighted $M S O$ logic over $\Delta$ and $K$ is given by the grammar

$$
\begin{aligned}
& \phi::=\operatorname{true}\left|P_{a}(x)\right| x \leq x^{\prime}|x \in X| \neg \phi|\phi \vee \phi| \exists x \cdot \phi \mid \exists X \cdot \phi \\
& \varphi::=k|\phi| \varphi \oplus \varphi|\varphi \otimes \varphi| \bigoplus_{x} \cdot \varphi\left|\bigoplus_{X} \cdot \varphi\right| \bigotimes_{x} \cdot \varphi \mid \bigotimes_{X} \cdot \varphi
\end{aligned}
$$

where $k \in K, a \in \Delta$.

We denote by $M S O(K, \Delta)$ the set of all weighted $M S O$ logic formulas $\varphi$ over $\Delta$ and $K$. We represent the semantics of formulas $\varphi \in M S O(K, \Delta)$ as series $\|\varphi\| \in K\left\langle\left\langle\Delta^{*}\right\rangle\right\rangle$. For the semantics of $M S O$ logic formulas $\phi$ we use the satisfaction relation as defined above. Therefore, the semantics of $M S O$ logic formulas $\phi$ gets only the values 0 and 1 .

Definition 5. Let $\varphi \in M S O(K, \Delta)$ and $\mathcal{V}$ be a finite set of variables with free $(\varphi) \subseteq$ $\mathcal{V}$. The semantics of $\varphi$ is a series $\|\varphi\|_{\mathcal{V}} \in K\left\langle\left\langle\Delta_{\mathcal{V}}^{*}\right\rangle\right\rangle$. Consider an element $(u, \sigma) \in \Delta_{\mathcal{V}}^{*}$. If $(u, \sigma) \notin N_{\mathcal{V}}$, then we let $\left(\|\varphi\|_{\mathcal{V}},(u, \sigma)\right)=0$. Otherwise, we define $\left(\|\varphi\|_{\mathcal{V}},(u, \sigma)\right) \in K$, inductively on the structure of $\varphi$, as follows:

- $\left(\|k\|_{\mathcal{V}},(u, \sigma)\right)=k$,

- $\left(\|\phi\|_{\mathcal{V}},(u, \sigma)\right)=\left\{\begin{array}{ll}1 & \text { if }(u, \sigma) \models \phi \\ 0 & \text { otherwise }\end{array}\right.$,

- $\left(\|\varphi \oplus \psi\|_{\mathcal{V}},(u, \sigma)\right)=\left(\|\varphi\|_{\mathcal{V}},(u, \sigma)\right)+\left(\|\psi\|_{\mathcal{V}},(u, \sigma)\right)$,

- $\left(\|\varphi \otimes \psi\|_{\mathcal{V}},(u, \sigma)\right)=\left(\|\varphi\|_{\mathcal{V}},(u, \sigma)\right) \cdot\left(\|\psi\|_{\mathcal{V}},(u, \sigma)\right)$,

- $\left(\left\|\bigoplus_{x} \cdot \varphi\right\|_{\mathcal{V}},(u, \sigma)\right)=\sum_{0 \leq i \leq n-1}\left(\|\varphi\|_{\mathcal{V} \cup\{x\}},(u, \sigma[x \rightarrow i])\right)$

- $\left(\left\|\bigoplus_{X} \cdot \varphi\right\|_{\mathcal{V}},(u, \sigma)\right)=\sum_{I \subseteq \operatorname{Dom}(u)}\left(\|\varphi\|_{\mathcal{V} \cup\{X\}},(u, \sigma[X \rightarrow I])\right)$,

- $\left(\left\|\bigotimes_{x} \cdot \varphi\right\|_{\mathcal{V}},(u, \sigma)\right)=\prod_{0 \leq i \leq n-1}\left(\|\varphi\|_{\mathcal{V} \cup\{x\}},(u, \sigma[x \rightarrow i])\right)$,

$-\left(\left\|\bigotimes_{X} \cdot \varphi\right\|_{\mathcal{V}},(u, \sigma)\right)=\prod_{I \subseteq D o m(u)}\left(\|\varphi\|_{\mathcal{V} \cup\{X\}},(u, \sigma[X \rightarrow I])\right)$.

We simply denote $\|\varphi\|_{\text {free }(\varphi)}$ by $\|\varphi\|$. If $\varphi$ is a sentence, then $\|\varphi\| \in K\left\langle\left\langle\Delta^{*}\right\rangle\right\rangle$. Furthermore, it holds [12]

$$
\left(\|\varphi\|_{\mathcal{V}},(u, \sigma)\right)=\left(\|\varphi\|,\left(u,\left.\sigma\right|_{\text {free }(\varphi)}\right)\right)
$$

for every $(u, \sigma) \in N_{\mathcal{V}}$.

Definition 6. A formula $\varphi \in M S O(K, \Delta)$ will be called restricted if 
- it contains no universal quantification of the form $\bigotimes_{X} \cdot \psi$, and

- whenever it contains a universal first order quantification $\bigotimes_{x} \cdot \psi$, then $\psi$ is a formula of the form $\psi=\oplus_{1 \leq i \leq n}\left(k_{i} \otimes \phi_{i}\right)$ where $k_{i} \in K$ and $\phi_{i}$ is an MSO logic formula for every $1 \leq i \leq n$. $\Delta)$

We denote with $R M S O(K, \Delta)$ the subclass of all restricted formulas in $M S O(K$,

Definition 7. A formula $\varphi \in R M S O(K, \Delta)$ is called restricted existential if it is of the form $\bigoplus_{X_{1}, \ldots, X_{n}} \cdot \psi$ with $\psi \in R M S O(K, \Delta)$ and $\psi$ contains no set quantification.

The subclass of all restricted existential formulas in $M S O(K, \Delta)$ is denoted by $\operatorname{REMSO}(K, \Delta)$.

Definition 8. A series $s$ over $\triangle$ and $K$ is called $M S O$ (resp. RMSO, REMSO) definable if there is sentence $\varphi$ in $M S O(K, \Delta)(\operatorname{resp} . \operatorname{RMSO}(K, \Delta), \operatorname{REMSO}(K, \Delta))$ such that $s=\|\varphi\|$.

Theorem 4. [12, 13] Let $K$ be a commutative semiring and $\Delta$ a finite alphabet. Then for every series $s \in K\left\langle\left\langle\Delta^{*}\right\rangle\right\rangle$ the following statements are equivalent.

i) $s$ is recognizable.

ii) $s$ is RMSO-definable.

iii) $s$ is REMSO-definable.

Furthermore, if the semiring $K$ is locally finite, then the above statements are also equivalent to

iv) $s$ is MSO-definable.

Now we are ready to introduce the $M S O$ logic characterization for series over the infinite alphabet $\Sigma$ and the commutative semiring $K$.

Definition 9. A series s over $\Sigma$ and $K$ is called VMSO (resp. VRMSO, VREMSO) definable if there is a subalphabet $\Gamma \subseteq_{\text {fin }} \Sigma$ and an MSO (resp. RMSO, REMSO) definable series $s^{\prime}$ over $\Delta=\Gamma \cup Z \cup\{y\}$ and $K$ such that

$$
(s, w)=\sum_{u \in \text { preim }_{\Delta}(w)}\left(s^{\prime}, u\right)
$$

for every $w \in \Sigma^{*}$.

Theorem 5. Let $K$ be a commutative semiring and $\Sigma$ an infinite alphabet. Then for every series $s \in K\left\langle\left\langle\Sigma^{*}\right\rangle\right\rangle$ the following statements are equivalent.

i) $s$ is v-recognizable.

ii) $s$ is VRMSO-definable. 
iii) $s$ is VREMSO-definable.

Furthermore, if the semiring $K$ is locally finite, then the above statements are also equivalent to

iv) $s$ is VMSO-definable.

Proof. We obtain our result by Theorem 4 and Definition 9, using similar arguments as the ones in the proof of Theorem 3.

\section{Weighted linear dynamic logic over infinite al- phabets}

Vardi in 2011 introduced a linear dynamic logic ( $L D L$ for short) over infinite words and stated the expressive equivalence of $L D L$ formulas to $\omega$-rational expressions (cf. [42]). $L D L$ is a combination of propositional dynamic logic and of classical $L T L$. In [20] the authors proved the coincidence of the classes of rational and $L D L$-definable languages interpreted over finite words. Recently, $L D L$ has been investigated in the quantitative setup for both finite and infinite words [16]. More precisely, the authors proved the expressive equivalence of weighted $L D L$ formulas to weighted automata for finite words over commutative semirings, and for infinite words over totally commutative complete semirings. In this section, we introduce a weighted $L D L$ over the infinite alphabet $\Sigma$ and the commutative semiring $K$, and we show the expressive equivalence of weighted $L D L$ formulas to weighted variable automata.

Let us firstly recall the basic definitions for weighted $L D L$ logic over finite alphabets [16]. Let $\Delta$ be a finite alphabet. For every letter $a \in \Delta$ we consider an atomic proposition $p_{a}$, and we let $P=\left\{p_{a} \mid a \in \Delta\right\}$. Moreover, for every $p \in P$ we identify $\neg \neg p$ with $p$.

Definition 10. The syntax of $L D L$ formulas $\psi$ over $\Delta$ is given by the grammar

$$
\begin{aligned}
& \psi::=\text { true }\left|p_{a}\right| \neg \psi|\psi \wedge \psi|\langle\theta\rangle \psi \\
& \theta::=\phi|\psi ?| \theta+\theta|\theta ; \theta| \theta^{+}
\end{aligned}
$$

where $p_{a} \in P$ and $\phi$ denotes a propositional formula over the atomic propositions in $P$.

For every $L D L$ formula $\psi$ and $u \in \Delta^{*}$ we define the satisfaction relation $u \models \psi$, inductively on the structure of $\psi$, as follows:

$$
\begin{aligned}
& -u \models \text { true, } \\
& \text { - } u \models p_{a} \text { iff } u(0)=a, \\
& -u \models \neg \psi \text { iff } u \not \models \psi, \\
& \text { - } u \models \psi_{1} \wedge \psi_{2} \text { iff } u \models \psi_{1} \text { and } u \models \psi_{2},
\end{aligned}
$$


- $u \models\langle\phi\rangle \psi$ iff $u \models \phi$ and $u_{\geq 1} \models \psi$,

- $u \models\left\langle\psi_{1}\right.$ ? $\rangle \psi_{2} \quad$ iff $u \models \psi_{1}$ and $u \models \psi_{2}$,

- $u \models\left\langle\theta_{1}+\theta_{2}\right\rangle \psi$ iff $u \models\left\langle\theta_{1}\right\rangle \psi$ or $u \models\left\langle\theta_{2}\right\rangle \psi$,

- $u \models\left\langle\theta_{1} ; \theta_{2}\right\rangle \psi$ iff $u=v v^{\prime}, v \models\left\langle\theta_{1}\right\rangle$ true, and $v^{\prime} \models\left\langle\theta_{2}\right\rangle \psi$,

- $u \models\left\langle\theta^{+}\right\rangle \psi$ iff there exists $n$ with $1 \leq n \leq|u|$ such that $u=\left\langle\theta^{n}\right\rangle \psi$, where $\theta^{n}, n \geq 1$ is defined inductively by $\theta^{1}=\theta$ and $\theta^{n}=\theta^{n-1} ; \theta$ for $n>1$.

Definition 11. The syntax of formulas $\varphi$ of the weighted $L D L$ over $\Delta$ and $K$ is given by the grammar

$$
\begin{aligned}
& \varphi::=k|\psi| \varphi \oplus \varphi|\varphi \otimes \varphi|\langle\rho\rangle \varphi \\
& \rho:=\phi|\varphi ?| \rho \oplus \rho|\rho \cdot \rho| \rho^{\oplus}
\end{aligned}
$$

where $k \in K, \phi$ denotes a propositional formula over the atomic propositions in $P$, and $\psi$ denotes an LDL formula as in Definition 10.

We denote by $L D L(K, \Delta)$ the set of all weighted $L D L$ formulas $\varphi$ over $\Delta$ and $K$. We represent the semantics $\|\varphi\|$ of the formulas $\varphi \in L D L(K, \Delta)$ as series over $\Delta$ and $K$.

Definition 12. Let $\varphi \in \operatorname{LDL}(K, \Delta)$. The semantics of $\varphi$ is a series $\|\varphi\| \in$ $K\left\langle\left\langle\Delta^{*}\right\rangle\right\rangle$. For every $u \in \Delta^{*}$ the value $(\|\varphi\|, u)$ is defined inductively as follows:

- $(\|k\|, u)=k$,

- $(\|\psi\|, u)=\left\{\begin{array}{ll}1 & \text { if } u \models \psi \\ 0 & \text { otherwise }\end{array}\right.$,

- $\left(\left\|\varphi_{1} \oplus \varphi_{2}\right\|, u\right)=\left(\left\|\varphi_{1}\right\|, u\right)+\left(\left\|\varphi_{2}\right\|, u\right)$

- $\left(\left\|\varphi_{1} \otimes \varphi_{2}\right\|, u\right)=\left(\left\|\varphi_{1}\right\|, u\right) \cdot\left(\left\|\varphi_{2}\right\|, u\right)$,

- $(\|\langle\phi\rangle \varphi\|, u)=(\|\phi\|, u) \cdot\left(\|\varphi\|, u_{\geq 1}\right)$,

- $\left(\left\|\left\langle\varphi_{1} ?\right\rangle \varphi_{2}\right\|, u\right)=\left(\left\|\varphi_{1}\right\|, u\right) \cdot\left(\left\|\varphi_{2}\right\|, u\right)$,

- $\left(\left\|\left\langle\rho_{1} \oplus \rho_{2}\right\rangle \varphi\right\|, u\right)=\left(\left\|\left\langle\rho_{1}\right\rangle \varphi\right\|, u\right)+\left(\left\|\left\langle\rho_{2}\right\rangle \varphi\right\|, u\right)$,

- $\left(\left\|\left\langle\rho_{1} \cdot \rho_{2}\right\rangle \varphi\right\|, u\right)=\sum_{\substack{v, v^{\prime} \in \Delta^{*} \\ u=v v^{\prime}}}\left(\left(\|\left\langle\rho_{1}\right\rangle\right.\right.$ true $\left.\left.\|, v\right) \cdot\left(\left\|\left\langle\rho_{2}\right\rangle \varphi\right\|, v^{\prime}\right)\right)$

- $\left(\left\|\left\langle\rho^{\oplus}\right\rangle \varphi\right\|, u\right)=\sum_{n \geq 1}\left(\left\|\left\langle\rho^{n}\right\rangle \varphi\right\|, u\right)$

where for the definition of $\left(\left\|\left\langle\rho^{\oplus}\right\rangle \varphi\right\|, u\right)$ we assume that $\|\langle\rho\rangle$ true $\|$ is proper, and $\rho^{n}, n \geq 1$ is defined inductively by $\rho^{1}=\rho$ and $\rho^{n}=\rho^{n-1} \cdot \rho$ for $n>1$. 
A series $s \in K\left\langle\left\langle\Delta^{*}\right\rangle\right\rangle$ is called $L D L$-definable if there is a formula $\varphi \in L D L(K, \Delta)$ such that $s=\|\varphi\|$.

Theorem 6. [16] Let $K$ be a commutative semiring and $\Delta$ a finite alphabet. A series $s \in K\left\langle\left\langle\Delta^{*}\right\rangle\right\rangle$ is recognizable iff it is LDL-definable.

Now we are ready to introduce the $L D L$ characterization for series over the infinite alphabet $\Sigma$ and the semiring $K$.

Definition 13. A series $s$ over $\Sigma$ and $K$ is called VLDL-definable if there is a subalphabet $\Gamma \subseteq_{\text {fin }} \Sigma$ and an $L D L$-definable series $s^{\prime}$ over $\Delta=\Gamma \cup Z \cup\{y\}$ and $K$ such that

$$
(s, w)=\sum_{u \in \text { preim }_{\Delta}(w)}\left(s^{\prime}, u\right)
$$

for every $w \in \Sigma^{*}$.

By Theorem 6 and Definition 13, using similar arguments as the ones in the proof of Theorem 3, we obtain the subsequent result.

Theorem 7. Let $K$ be a commutative semiring and $\Sigma$ an infinite alphabet. Then a series $s \in K\left\langle\left\langle\Sigma^{*}\right\rangle\right\rangle$ is v-recognizable iff it is VLDL-definable.

\section{Application to variable finite automata}

In this section, we derive new results for the class of languages accepted by variable finite automata (vfa for short) over the infinite alphabet $\Sigma$ (cf. [21, 22]). We need first to recall the definition of vfa from [21, 22] but we follow the terminology used previously for wva. Let $Z$ be a finite set of bounded variables and $y$ a free variable. Then, a variable finite automaton over $\Sigma$ is a pair $\mathcal{A}=\langle\Sigma, A\rangle$ where $A=\left(Q, \Gamma_{A}, I, E, F\right)$ is a finite automaton with input alphabet $\Gamma_{A}=\Sigma_{A} \cup Z \cup\{y\}$ where $\Sigma_{A} \subseteq$ fin $\Sigma$. The language of $\mathcal{A}$ is defined by

$$
L(\mathcal{A})=\bigcup_{\substack{u \in L(A) \\ h \in V R\left(\Gamma_{A}\right)}} h(u) .
$$

Then the vfa $\mathcal{A}=\langle\Sigma, A\rangle$ can be considered, in the obvious way, as a wva $\mathcal{A}^{\prime}$ over the Boolean semiring $\mathbb{B}$. Moreover, it holds

$$
w \in L(\mathcal{A}) \quad \text { iff } \quad\left(\left\|\mathcal{A}^{\prime}\right\|, w\right)=1
$$

for every $w \in \Sigma^{*}$.

A language $L \subseteq \Sigma^{*}$ is called recognizable if there is a vfa $\mathcal{A}=\langle\Sigma, A\rangle$ such that $L=L(\mathcal{A})$.

Theorem 8. Let $\Sigma$ be an infinite alphabet. The class of recognizable languages over $\Sigma$ is closed under union, intersection, concatenation, Kleene star, and shuffle product. $^{5}$

\footnotetext{
${ }^{5}$ The closure under union and intersection has been also proved in $[21,22]$.
} 
Proof. In Theorem 1 we let $K=\mathbb{B}$. Then for every $L \subseteq \Sigma^{*}$, clearly $L$ is recognizable iff its characteristic series $1_{L} \in \mathbb{B}\left\langle\left\langle\Sigma^{*}\right\rangle\right\rangle$ is v-recognizable. We conclude our result by the idempotency property of $\mathbb{B}$.

Next we define the notions of rational (resp. $M S O$-definable, $L D L$-definable) languages over the infinite alphabet $\Sigma$.

Definition 14. Let $\Sigma$ be an infinite alphabet. A language $L$ over $\Sigma$ is called rational (resp. MSO-definable, $L D L$-definable) if there is a subalphabet $\Gamma \subseteq_{\text {fin }} \Sigma$ and a rational (resp. MSO-definable, LDL-definable) language $L^{\prime}$ over $\Delta=\Gamma \cup Z \cup\{y\}$ such that

$$
L=\bigcup_{\substack{u \in L^{\prime} \\ h \in V R(\Delta)}} h(u)
$$

The next theorem establishes new characterizations for the class of languages accepted by vfa.

Theorem 9. Let $\Sigma$ be an infinite alphabet and $L \subseteq \Sigma^{*}$. Then the following statements are equivalent.

i) $L$ is recognizable.

ii) L is rational.

iii) $L$ is MSO-definable.

iv) $L$ is LDL-definable.

Proof. We take into account the definition of recognizable languages over the infinite alphabet $\Sigma$ and Definition 14 . Then we obtain the equivalence $(i) \Longleftrightarrow($ ii $)$ by Kleene's theorem, the equivalence $(i) \Longleftrightarrow($ iii $)$ by Büchi's theorem, and the equivalence $(i) \Longleftrightarrow(i v)$ by $[20]$.

\section{Conclusion}

We introduced weighted variable automata over an infinite alphabet $\Sigma$ and a commutative semiring $K$. Our model is based on the variable automaton model of $[21,22]$ but we followed the terminology used in [26, 27] for variable tree automata over infinite ranked alphabets. Indeed, that terminology presents in a strict mathematical way the operation of (weighted) variable automata models. With the additional assumption that $K$ is idempotent we proved the closure of the class of series accepted by our models under the operations of sum, and scalar, Hadamard, Cauchy, and shuffle products, as well as star operation applied to proper series. We introduced the notion of rational series over the infinite alphabet $\Sigma$ and an arbitrary semiring $K$ and stated a Kleene-Schützenberger type theorem. We defined a weighted $M S O$ logic over the infinite alphabet $\Sigma$ and the commutative semiring $K$ 
and proved a Büchi type theorem, extending the work of Droste and Gastin [12, 13] to series over infinite alphabets. Finally, we considered a weighted $L D L$ over the infinite alphabet $\Sigma$ and the commutative semiring $K$ and proved the expressive equivalence of its formulas to weighted variable automata.

It is an open problem whether we can relax the idempotency property of the semiring $K$ for the closure properties of the class $V \operatorname{Rec}(K, \Sigma)$. Furthermore, it should be very interesting to study the weighted variable automata theory over more general weight structures, contributing to real world applications, like for instance over valuation monoids [15].

\section{References}

[1] P. Barceló, J. Reutter, L. Libkin, Parameterized regular expressions and their languages, Theoret. Comput. Sci. 474(2013) 21-45.

[2] W. Belkhir, Y. Chevalier, M. Rusinowitch, Fresh-variable automata: Application to service composition, in: Proceedings of SYNASC 2013, pp.153-160.

[3] W. Belkhir, Y. Chevalier, M. Rusinowitch, Guarded variable automata over infinite alphabets, CoRR abs/1304.6297, 2013.

[4] M. Benedikt, C. Ley, G. Puppis, Automata vs. logics on data words, in: Proceedings of CSL 2010, LNCS 6247(2010) 110-124.

[5] M. Bojańczyk, C. David, A. Muscholl, T. Schwentick, L. Segoufin, Twovariable logic on data words, ACM Trans. Comput. Log. 12(4)(2011) 27.

[6] B. Bollig, P. Habermehl, M. Leucker, B. Monmege, A robust class of data languages and an application to learning, Log. Methods in Comput. Sci. $10(4)(2014) 19$.

[7] J.R. Büchi, Weak second-order arithmetic and finite automata, Z. Math. Logik Grundlager Math. 6(1960) 66-92.

[8] P. Černý, S. Gopi, T.A. Henzinger, A. Radhakrishna, N. Totla, Synthesis from incompatible specifications, in: Proceedings of EMSOFT 2012, ACM (2012) $53-62$.

[9] J. Dassow, G. Vaszil, P finite automata and regular languages over countably infinite alphabets, in: Proceedings of WMC 7, LNCS 4361(2006) 367-381.

[10] P. Degano, G.L. Ferrari, G. Mezzetti, Nominal automata for resource usage control, in: Proceedings of CIAA 2012, LNCS 7381(2012) 125-137.

[11] A. Deharbe, F. Peschanski, The omniscient garbage collector: A resource analysis framework, in: Proceedings of ACSD 2014, IEEE Computer Society 102111. 
[12] M. Droste, P. Gastin, Weighted automata and weighted logics, Theoret. Comput. Sci. 380 (2007) 69-86.

[13] M. Droste, P. Gastin, Weighted automata and weighted logics, chapter 5, in [14].

[14] M. Droste, W. Kuich, H. Vogler (eds), Handbook of Weighted Automata. EATCS Monographs in Theoretical Computer Science, Springer, Berlin, 2009.

[15] M. Droste, I. Meinecke, Weighted automata and regular expressions over valuation monoids, Internat. J. Found. Comput. Sci. 22(2011) 1829-1844.

[16] M. Droste, G. Rahonis, Weighted linear dynamic logic, in: Proceedings of GandALF 2016, EPTCS 226(2016) 149-163.

[17] C. Elgot, Decision problems of finite automata design and related arithmetics, Trans. Amer. Math. Soc. 98(1961) 21-52.

[18] Z. Ésik, W. Kuich, Finite automata, chapter 3, in [14].

[19] P. Gastin, B. Monmege, A unifying survey on weighted logics and weighted automata, Soft Computing, to appear.

[20] G. De Giacomo, M. Vardi, Linear temporal logic and linear dynamic logic on finite traces, in: Proceedings of IJCAI, IJCAI/AAAI. Available at http://www.aaai.org/ocs/index.php/IJCAI/IJCAI13/paper/view/6997

[21] O. Grumberg, O. Kupferman, S. Sheinvald, Variable automata over infinite alphabets, in: Proceedings of LATA 2010, LNCS 6031(2010) 561-572.

[22] O. Grumberg, O. Kupferman, S. Sheinvald, Variable automata over infinite alphabets, http://www.cs.huji.ac.il/ ornak/publications/lata10.pdf

[23] O. Grumberg, O. Kupferman, S. Sheinvald, Model checking systems and specifications with parameterized atomic propositions, in: Proceedings of ATVA 2012, LNCS 7561(2012) 122-136.

[24] M. Kaminski, N. Francez, Finite-memory automata, Theoret. Comput. Sci. 134(1994) 329-363.

[25] M. Kaminski, T. Tan, Regular expressions for languages over infinite alphabets, Fund. Inform. 69(2006) 301-318.

[26] I.-E. Mens, Tree Automata over Infinite Ranked Alphabets, Master thesis, Thessaloniki 2011, http://invenio.lib.auth.gr/record/128884/files/GRI-20128361.pdf

[27] I.-E. Mens, G. Rahonis, Variable tree automata over infinite ranked alphabets, in: Proceedings of CAI 2011, LNCS 6742(2011) 247-260. 
[28] F. Neven, T. Schwentick, V. Vianu, Towards regular languages over infinite alphabets, in: Proceedings of MFCS 2001, LNCS 2136(2001), 560-572.

[29] F. Neven, T. Schwentick, V. Vianu, Finite state machines for strings over infinite alphabets, ACM Trans. Comput. Log. 5(2004) 403-435.

[30] G. Pellegrino, Q. Lin, C. Hammerschmidt, S. Verwer, Learning deterministic finite automata from infinite alphabets, in: Proceedings of ICGI 2016, JMLR: Workshop and Conference Proceedings 57(2016) 120-131.

[31] M. Pittou, Weighted Variable Automata over Infinite Alphabets, Master Thesis, Thessaloniki 2014, http://ikee.lib.auth.gr/record/135664/files/GRI-201513622.pdf

[32] M. Pittou, G. Rahonis, Weighted variable automata over infinite alphabets, in: Proceedings of CIAA 2014, LNCS 8587(2014) 304-317.

[33] M. Praveen, B. Srivathsan, Nesting depth of operators in graph database queries: Expressiveness vs evaluation complexity, in: Proceedings of ICALP 2016, LIPIcs 55(2016), 117:1-117:14.

[34] J. Sakarovitch, Elements of Automata Theory, Cambridge University Press, 2009.

[35] M. Schützenberger, On the definition of a family of automata, Information and Control 4(1961) 245-270.

[36] L. Segoufin, Automata and logics for words and trees over an infinite alphabet, in: Proceedings of CSL 2006, LNCS 4207(2006) 41-57.

[37] Y. Shemesh, N. Francez, Finite-state unification automata and relational languages, Inform. and Comput. 114(1994) 192-213.

[38] F. Song, Z. Wu, On temporal logics with data variable quantifications: Decidability and complexity, Inform. and Comput. 251(2016) 104-139.

[39] T. Tan, Graph reachability and pebble automata over infinite alphabets, ACM Trans. Comput. Log. 14(3)(2013) 19:1-19:31.

[40] W. Thomas, Languages, automata and logic, in: Handbook of Formal Languages vol. 3 (G. Rozenberg, A. Salomaa, eds.), Springer, 1997, pp. 389-485.

[41] B. Trakhtenbrot, Finite automata and logic of monadic predicates (in Russian), Doklady Akademii Nauk SSSR 140(1961) 326-329.

[42] M.Y. Vardi, The rise and fall of LTL, in: Proceedings of GandALF 2011, EPTCS 54(2011).

[43] D. Vrgoč, Using variable automata for querying data graphs, Inf. Process. Lett. 115(3)(2015) 425-430. 